\title{
A mechanistic model to predict droplet drying history and particle shell formation in multicomponent systems
}

DOI:

10.1016/j.ces.2020.115713

\section{Document Version}

Accepted author manuscript

Link to publication record in Manchester Research Explorer

\section{Citation for published version (APA):}

Abdullahi, H., Burcham, C. L., \& Vetter, T. (2020). A mechanistic model to predict droplet drying history and particle shell formation in multicomponent systems. Chemical Engineering Science, 115713.

https://doi.org/10.1016/j.ces.2020.115713

\section{Published in:}

Chemical Engineering Science

\section{Citing this paper}

Please note that where the full-text provided on Manchester Research Explorer is the Author Accepted Manuscript or Proof version this may differ from the final Published version. If citing, it is advised that you check and use the publisher's definitive version.

\section{General rights}

Copyright and moral rights for the publications made accessible in the Research Explorer are retained by the authors and/or other copyright owners and it is a condition of accessing publications that users recognise and abide by the legal requirements associated with these rights.

\section{Takedown policy}

If you believe that this document breaches copyright please refer to the University of Manchester's Takedown Procedures [http://man.ac.uk/04Y6Bo] or contact uml.scholarlycommunications@manchester.ac.uk providing relevant details, so we can investigate your claim.

\section{OPEN ACCESS}




\title{
A mechanistic model to predict droplet drying history and particle shell formation in multicomponent systems
}

\author{
Hassan Abdullahi ${ }^{\mathrm{a}}$, Christopher L. Burcham ${ }^{\mathrm{b}}$, Thomas Vetter ${ }^{\mathrm{a}, *}$ \\ ${ }^{a}$ University of Manchester, Department of Chemical Engineering and Analytical Science, M13 9PL Manchester, United \\ Kingdom \\ ${ }^{b}$ Eli Lilly and Company, Indianapolis, USA
}

\section{Abstract}

Spray drying allows tuning the physical properties of the resulting powders widely. However, targeted process design is complicated by the interplay between process characteristics and the non-ideal physical properties exhibited by multicomponent mixtures, such as pharmaceutical formulations. This work presents a mechanistic model describing the drying of single droplets. The model includes heat and mass balances, non-ideal vapour-liquid equilibria, and population balances describing the evolution of particulates within the drying droplet. The model is applied to (up to) ternary mixtures of solvents, polymers and solutes, and predicts properties of the drying droplet such as the time of shell formation and the size of the particle obtained after drying. A comparison with experimental data from single droplet drying experiments carried out at defined relative vapour saturation and temperature shows that the model can be used to predict shell formation (as well as other properties) for the systems studied here.

8 Keywords: spray drying; single droplet drying; acoustic levitation; non-ideal multicomponent 9 systems; mechanistic population balance equation model

\footnotetext{
${ }^{*}$ Corresponding author: phone $+44161306-4370$.

Email address: thomas.vetter@manchester.ac.uk (Thomas Vetter)
} 
The concept of engineering materials to improve functionality is widely applied in many industries where relationships between structure and functional properties are of vital importance $[1,2]$. In the pharmaceutical industry, formulating active pharmaceutical ingredients (APIs) often requires physiochemical protection of the API and API release modulation in addition to chemical/physical modifications for efficient drug delivery. As such, the development of highly engineered micro and nanoparticle matrices has become critical for use in drug delivery technologies [3, 4]. Spray drying offers an opportunity to design such particles in a single step involving simultaneous drying and particle formation from droplets containing API, excipient and solvent either in the form of a solution or a suspension. It has gained wide acceptance in the pharmaceutical industry as an effective method for the production of amorphous API-excipient matrices due to the rapid evaporation of solvent, thereby directly increasing the dissolution rate of many insoluble drugs [3,5-7]. Spray drying involves first the atomisation of a liquid or suspension into droplets, typically with sizes between 10 and $100 \mu \mathrm{m}$, followed by solvent evaporation through convective heating leading to particle formation and subsequent drying. The large surface area created by atomisation allows the drying process and particle formation to occur rapidly and simultaneously $[7,8]$.

By controlling process conditions and formulation properties, the particles produced can be typically tailored for use in oral and pulmonary drug delivery systems. Each of these applications requires particles with different physical characteristics such as size, bulk density and porosity $[9,10]$. Predicting such product characteristics is difficult due to the strong coupling between atomisation, drying and particle formation [7]. In addition, particle characteristics can impact the ease at which post-processing stages such as tableting are accomplished and as such, a deeper understanding of the processes leading to particle formation is required. In general, experimental investigations carried out at scale can be time-consuming and material intensive. Therefore, developing appropriate modelling methodologies to predict such varying product characteristics can be highly valuable.

Theoretical models that predict particle morphologies and process behaviour require an adequate description of the drying kinetics of droplets, which in turn become wet and then dry particles. In order to validate such models, access to insightful experimental data is required. However, it is difficult to 
precisely follow the evolution of droplets/particles in an actual spray drying process, because there are typically millions of rapidly drying droplets simultaneously present in the spray drying chamber. Therefore, several attempts have been made to develop comparable experimental and theoretical methods based on single droplet drying (SDD) approaches. Although, inter-droplet effects are ignored in SDD methods, they are useful to understand process behaviour and for validation of modelling methodologies [11, 12]. Experimental and model attempts have been described and summarised in several review articles [11, 13-17]. Although several modelling methodologies have been well described, they are still limited, particularly with respect to their treatment of non-ideal and multicomponent systems. The droplet drying process is often separated into two main drying regimes. The first drying stage, where the droplet is still completely covered with the liquid phase and the second drying stage where a solid shell is present. In reality, formulations subjected to spray drying are often complex multicomponent systems. A detailed overview of theoretical methods is given in a review by Mezhericher et al. [18]. Until now, the most advanced of these combine mass and energy balances with a population balance approach to describe the entire droplet drying history; including the mechanisms of particle formation (nucleation and growth) [19-21]. This allows for a complete mechanistic description of the processes occurring within the droplet domain. Such models thus require the solution of a set of partial differential equations describing heat and mass transport as well as fluid mechanics within a moving domain. Although this is computationally intensive, the approach can capture the main drying behaviour. Seydel et al. [21] described a binary solution using a 1D population balance model to describe the crystallisation of individual particles within the drying droplet. This was combined with equations to describe the evolution of concentration and droplet surface temperature during drying. The model was based on several assumptions that includes ideal vapour-liquid equilibrium and spherical symmetry of the droplet. In the second drying stage, the critical point of the shell was identified at a predefined solid fraction at the droplet surface and drying was assumed to proceed in a funicular manner (dryshell route) where the evaporation front recedes into the recess of the formed particle. Experimental results were used to validate the physical processes leading to particle formation and a good agreement was obtained. More recently, Handscomb et al. [19, 20] extended the model of Seydel et al. [21] to predict the structural evolution of an ideal binary droplet containing suspended solids. The continuous and discrete phases were similarly described, with the critical point identified by a predetermined solid volume fraction. The droplet temperature was assumed to be uniform in both drying stages. The model 
however included a shell thickening regime such that droplet shrinkage can continue from the point at which the initial shell is formed to when the shell becomes strong enough to withstand external pressure exerted on the shell surface. This was coupled with a known dry shell model, and a model involving the presence of a bubble (wet shell). It has been postulated that such sub-models for the second drying stage (wet shell, dry shell, etc.) present the key to developing a comprehensive model for spray dryers $[10,22]$. The drying history prior to shell formation has been shown to determine events leading to the use of the several sub-models [22]. Although previous researchers have developed powerful insight into describing particle morphologies, the models until now have limited validation and applicability, particularly in the context of complex multicomponent formulations.

In the present work, an extensive evaporation and particle formation model is used to describe drying prior to shell formation in multicomponent systems. The model incorporates population balance equations to describe the evolution of the particle size distribution (PSD) within each part of the domain. Unlike previous models where shell formation is activated through a predetermined solid fraction, information obtained from the PSD is used to describe shell formation directly. The assumptions of uniform droplet temperature is relaxed. Although, Farid [23] shows that this assumption is appropriate during the initial phase of drying, the distribution during the later phase of drying (following shell formation), or in systems where thermal conductivity in different phases varies by some order of magnitude cannot be ignored. The evaporation phenomenon is described in a more sophisticated manner than in previous works [19-21], accounting for non-ideality of mixtures and the effect of Stefan flow (due to moving droplet boundary) within the droplet. Overall, the model combines some features of previous works in this area, but it is extended to multicomponent mixtures with considerable non-ideality in order to describe typical pharmaceutical formulations. In the work that follows, a model describing the drying process up to the point of shell formation (i.e., the critical point) is discussed. A model for the second drying stage, i.e., after shell formation, will form the subject of future work. The remainder of this work is structured as follows: in Section 2, a theoretical understanding of droplet drying kinetics and particle formation is explored. In Section 3, model equations are formulated that describe transport of mass and energy within the droplet and between the droplet and gas phase, as well as the growth and nucleation of individual particles within the droplet. In Section 4, the numerical implementation of the moving boundary problem is discussed. In Section 5, the experimental methods used for model 
validation are discussed and in Section 6, the effect of drying condition on droplet drying kinetics is discussed in detail and relevant conclusions are made in Section 7.

\section{Drying process and particle morphologies}

Before entering into the description of the model, it is useful to summarise the physics and mechanisms involved in droplet drying. Ranz and Marshall [24] and Charlesworth and Marshall [25] provided a detailed experimental and theoretical basis for the drying history of droplets containing solutes. Initially, concentration gradients are established within the droplet due to solvent evaporation at the droplet surface. This leads to solvent migrating towards the surface and solutes moving towards the centre due to diffusion. The extent of the gradient is the result of the drying kinetics ensuing at different temperature, relative saturation of the gas phase and composition of the liquid phase. The drying history of a droplet containing a solution can be well summarised with a temperature versus time curve (Figure 1) [26]. In the initial stage of drying, the droplet is rapidly heated to the wet bulb temperature (A-B). As long as the droplet surface is saturated with moisture the temperature remains constant with drying proceeding at a near constant rate (B-C). This period of drying is easily identified by the shrinking of droplet diameter. At the critical point there is insufficient moisture to maintain the surface saturation, the temperature starts to rise until it reaches the boiling point of the liquid (C-D) where vaporisation (above boiling point) begins. At this point, ideal shrinkage ceases. This phase coincides with the formation of a shell/crust at the outer layer of the droplet, and drying changes from being surface evaporation controlled to being controlled by internal moisture migration. In Figure 1, a shell is formed somewhere between point $\mathrm{C}$ and D. During boiling, a considerable amount of energy is required in the form of latent heat of vaporisation, therefore the sensible heating of the droplet/particle halts and drying is controlled solely by external heat transfer (D-E). Once the moisture removal is complete, the temperature of the particle rises until it thermally equilibrates with the surrounding gas temperature $[26,27]$. In many industrial drying processes, drying is often terminated before point $\mathrm{D}$ (i.e., boiling of the solvent) since this necessitates significant energy requirements as well as appropriate process residence times. Depending on the droplet drying history, a range of particle morphologies can be obtained at the end of drying [28]. Several authors have given an account of the complex intra-droplet transport phenomena using single droplet drying studies. This has led to the classification of different particle morphologies 


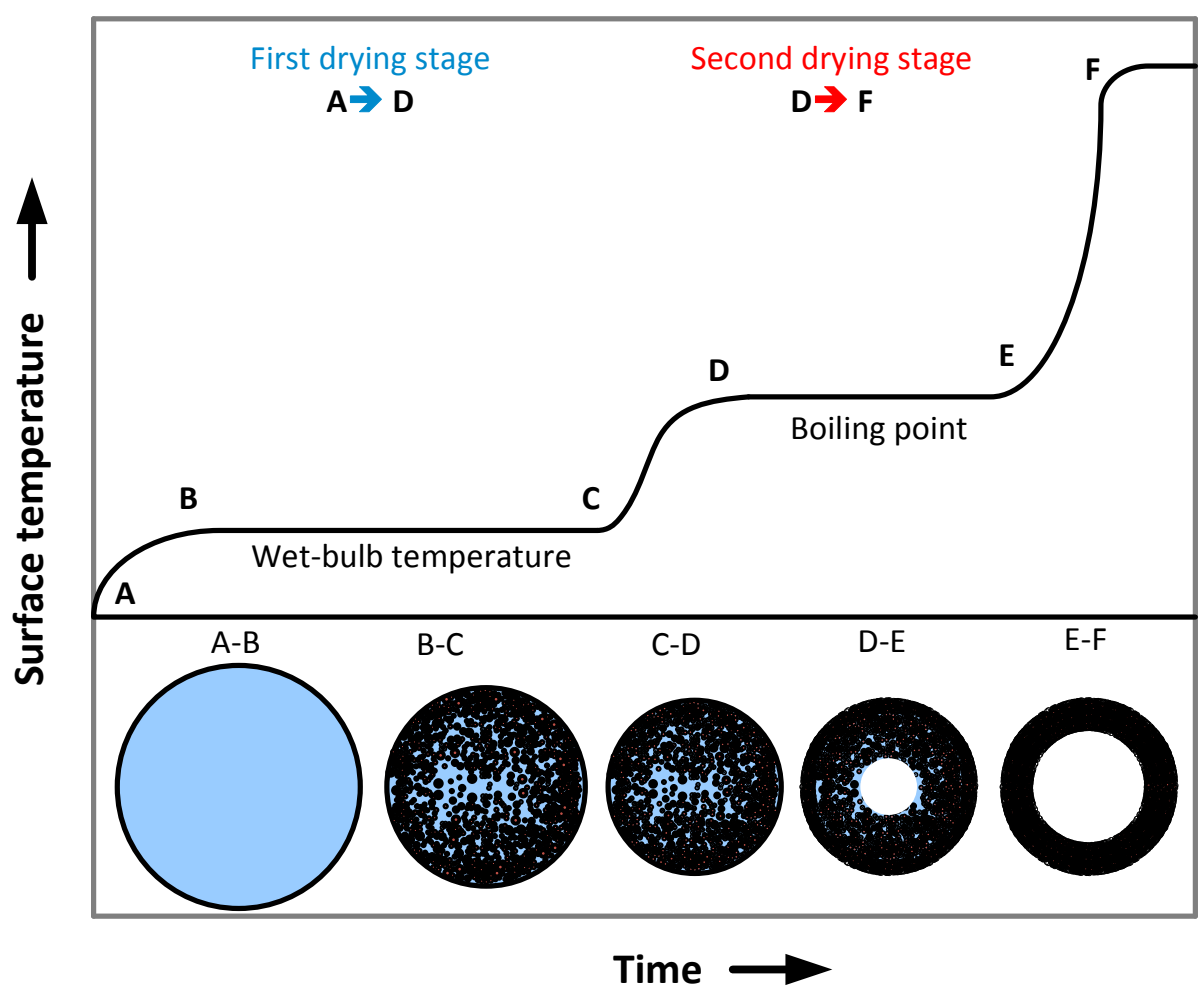

Figure 1: A typical droplet/particle surface temperature profile. At the bottom, one characteristic type of particle morphology is shown; an overview of other attainable morphologies is given in Figure 2.

obtainable when droplets are spray dried $[10,28]$. The different obtainable particle morphologies are illustrated in Figure 2. When drying is slow, there is sufficient time for the equilibration of concentration gradients. In this case the Peclet number, Pe defined as the ratio of convective to diffusive transport is small. This leads to a (close to) uniform droplet and eventually a solid rigid porous structure. When drying is fast (i.e. large Peclet number), there is little time for diffusion to occur, leading to large concentration gradients between the surface and the centre of the droplet. This may lead to the formation of a variety of complex morphologies for the dried particle. For instance, the formation of a vapour bubble within the droplet can occur due to sudden pressure gradients. This can lead to a cycle of inflation and deflation of the forming particle and subsequently formation of rigid hollow structures. In such a case, particles may also deform to produce structures that are cracked or shrivelled (Figure 2). 


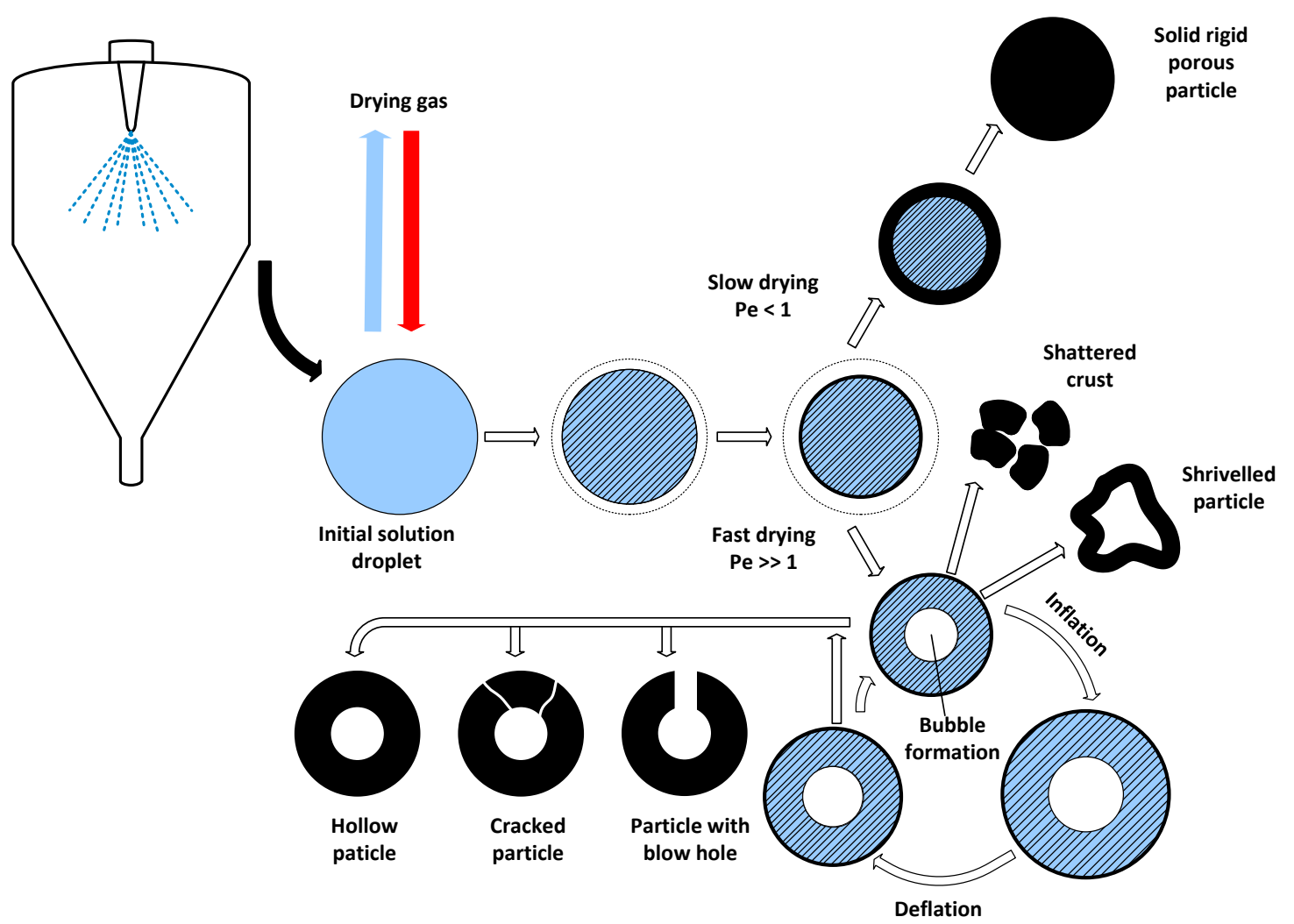

Figure 2: Examples of possible morphological evolutions of a solution droplet during drying. This work focuses on the evolution of the droplet/ particle up to the point where the shell is formed.

\section{Model Formulation}

\subsection{Prior to Shell formation}

In the drying of a droplet containing two or more species of dissolved solutes, the solute molecules move simultaneously relative to each other by advection and diffusion in the continuous liquid phase, as the droplet shrinks due to solvent evaporation. This leads to concentration gradients, with particularly high concentrations occurring at the droplet surface. If the concentration exceeds the solubility limit, i.e., is higher than the equilibrium concentration at a given temperature and pressure, a driving force for crystallisation exists. This driving force, commonly expressed as supersaturation, i.e., the ratio of actual concentration and the equilibrium concentration at a given time and position, leads to nucleation (increase in number) and growth (increase in size) of particulates in the solution. This in turn leads to a rapid increase in the total solid fraction of particulates within the droplet, which ultimately leads to the 

problem to be solved is therefore a two-dimensional one (in particle size and in radial coordinate).

formation of a shell.

The following section will introduce equations governing mass and energy transport in the liquid and solid phases. To simplify the description of these transport processes, the droplet is assumed to be spherically symmetric during the entire drying duration. This reduces the problem to a one-dimensional problem in spherical coordinates. It can be further assumed that the evolving discrete phase can be described by a single dimension (particle size) where particles form at small size and grow to larger sizes over time. This description naturally leads to describing the solid phase with a particle size distribution that evolves over time and is different at different radial positions in the droplet. The overall
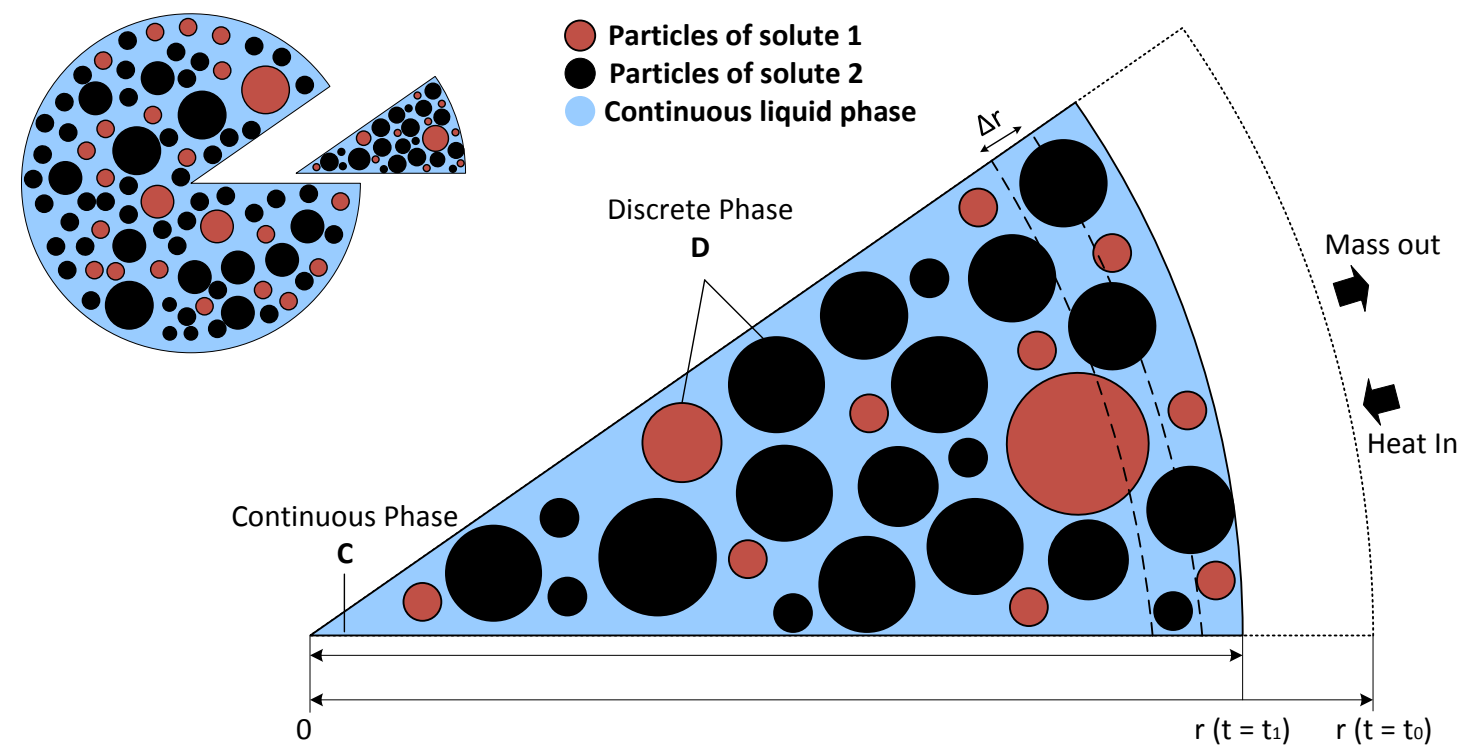

Figure 3: Illustration of the control volume (as used in the model) within a shrinking droplet during the first drying stage.

\subsubsection{Continuous phase model}

To formulate the model, a control volume of a droplet is shown in Figure 3. Many previous models have assumed that internal transport within a droplet is dominated by diffusion. However, this is not entirely true as diffusion in concentrated systems can cause the bulk motion of fluid. The overall velocity of molecules undergoing mass transport will incorporate both advection and diffusion and hence, a suitable choice of reference velocity must be considered to determine the advective flux caused by 
diffusion [29]. Writing a mass conservation equation on the control volume shown in Figure 3, the evolution of specie concentration in the droplet over time can be described;

$$
\frac{\partial c_{i}}{\partial t}=-\frac{1}{r^{2}} \frac{\partial}{\partial r}\left(r^{2} u^{\langle C\rangle} c_{i}\right)+\frac{1}{r^{2}} \frac{\partial}{\partial r}\left(r^{2} \sum_{j=1}^{n-1} D_{i j}^{\langle C\rangle} \frac{\partial c_{j}}{\partial r}\right)-\rho_{i}^{\langle D\rangle} \frac{\partial \varepsilon_{i}}{\partial t}
$$

where $i=1,2, \ldots, n-1$ for an $n$ component system. The first and second term on the right hand side are mass transport terms due to advection and diffusion, respectively, while the last term describes the mass of solute consumed for nucleation and growth of particles from the solution. $c_{i}$ denotes the concentration of component $i$, defined by $c_{i}=\rho^{\langle C\rangle} w_{i}(1-\varepsilon) . \rho^{\langle C\rangle}$ is the density of the continuous phase, $w_{i}$ is the mass fraction of component $i$ in the continuous phase, $\varepsilon$ is the solid fraction, $t$ is time, $r$ is the spatial radial coordinate, $u$ is the diffusion dependent advective velocity and $D_{i j}$ are multicomponent diffusion coefficients. This equation is valid from the centre of the droplet $(r=0)$ to the surface of the droplet $\left(r=r_{\mathrm{s}}\right)$. The value of $\varepsilon$ can be obtained from:

$$
\frac{\partial \varepsilon_{i}}{\partial t}=k_{v i} \frac{\partial \mu_{3 i}}{\partial t}=k_{v i} \frac{\partial}{\partial t} \int_{0}^{\infty} N_{i} L^{3} d L
$$

where $k_{v}$ is a shape factor relating the volume of a particle to the cube of its characteristic size. This means that $k_{v}=1$ for cubes, $k_{v}=\pi / 6$ for spheres and $k_{v} \ll 1$ for needles (when the characteristic size of the needles is their length). $\mu_{3}$ is the third moment of the PSD, $\rho_{i}^{\langle D\rangle}$ is the density of the crystal, $N_{i}$ is the PSD (number of particles per total volume per particle size) and $L$ is the characteristic size (length) of the particles.

The following dependencies of the different quantities exist:

$$
\mathbf{c}=\mathbf{c}(t, r), \boldsymbol{\rho}^{\langle C\rangle}=\boldsymbol{\rho}^{\langle C\rangle}(c, T), \mathbf{T}=\mathbf{T}(t, r), \boldsymbol{\varepsilon}=\boldsymbol{\varepsilon}(t, r)
$$

$$
\mathbf{u}=\mathbf{u}(c), \mathbf{D}=\mathbf{D}(\eta, T) \boldsymbol{\eta}=\boldsymbol{\eta}(c, T) \mathbf{N}=\mathbf{N}(t, r, L)
$$

where $\eta$ is the dynamic viscosity of the liquid and $T$ is the droplet temperature.

In general, the diffusion coefficients in a multicomponent system are not symmetrical (i.e. $D_{i j} \neq$ $\left.D_{j i}\right)$. This implies that the flux of one component is related to the concentration gradient of $(n-1)$ 
solutes, where one component is designated as the solvent. Hence in an $n$-component system, $(n-1)^{2}$ independent diffusion coefficients are required to describe the system, where the value of the main coefficients $\left(D_{i j, i=j}\right)$ are similar to that in a binary solution. The cross coefficients $\left(D_{i j, i \neq j}\right)$ can however be significantly lower. Values of these diffusion coefficients are preferably obtained experimentally. In order to write an expression for the diffusion dependent advective velocity, the volume fluxes are assumed to be as in an ideal system and the volume average advective velocity can be written in terms of specie concentration as [29]:

$$
u^{\langle C\rangle}=D_{12} \frac{\rho_{1}-\rho_{2}}{\rho_{1} \rho_{2}-\rho_{2} c_{1}+\rho_{1} c_{1}} \frac{\partial c_{1}}{\partial r}
$$

Eq. (1) is complemented by the following boundary conditions:

Symmetry is assumed at the centre of the droplet

$$
\frac{\partial c_{i}}{\partial r}=0 \quad \text { at } \quad r=0
$$

and a defined evaporative flux at the droplet surface

$$
-u^{\langle C\rangle} c_{i}+\sum_{j=1}^{n-1} D_{i j}^{\langle C\rangle} \frac{\partial c_{j}}{\partial r}=\delta_{i} \frac{\dot{m}_{\mathrm{v}, i}}{4 \pi r^{2}} \quad \text { at } \quad r=r_{s}
$$

where $\delta_{i}$ is 1 for the evaporating component(s), 0 for the non-evaporating component(s) in Eq. (4b), and $\dot{m}_{\mathrm{v}, i}$ denotes the mass vaporisation rate of component $i$.

\subsubsection{Discrete phase model}

In a similar manner, a conservation equation can be written to describe the evolution of particles within the control volume. The solid phase evolves in the spatial domain through advection/diffusion and in the internal particle length coordinate through nucleation/growth. The change in number density of particles resulting from this can be written as:

$$
\frac{\partial N_{i}}{\partial t}=-\frac{1}{r^{2}} \frac{\partial}{\partial r}\left(r^{2} u^{\langle D\rangle} N_{i}\right)+\frac{1}{r^{2}} \frac{\partial}{\partial r}\left(r^{2} \sum_{j=1}^{n-1} D_{i j}^{\langle D\rangle} \frac{\partial N_{j}}{\partial r}\right)-\frac{\partial\left(G_{i} N_{i}\right)}{\partial L}
$$


205

208

where $G$ is the particle growth rate (i.e., the increase in the size of the particulates with time) and is a function of the supersaturation of the solution.

Eq. (5) exists in two coordinates and thus the solution to the partial differential equation requires two boundary conditions each for the radial and particle size coordinates (for each particle forming component). In the radial coordinate, $r$ the boundary condition is that of symmetry and no flux of particles at the droplet centre and surface respectively:

$$
\frac{\partial N_{i}}{\partial r}=0 \quad \text { at } \quad r=0
$$

$$
-u^{\langle D\rangle} N_{i}+\sum_{j=1}^{n-1} D_{i j}^{\langle D\rangle} \frac{\partial N_{j}}{\partial r}=0 \quad \text { at } \quad r=r_{s}
$$

In the particle size coordinate, $L$ the first boundary condition accounts for the nucleation of particles. The second condition is that no infinitely sized particles exist. This is written mathematically as:

$$
G_{i} N_{i}(t, r, L)=J_{i} \quad \text { at } \quad L=L_{\min }
$$

$$
N_{i}(t, r, L)=0 \quad \text { at } \quad L=\infty
$$

The rate of nucleation $J$ and subsequent growth $G$ of the crystals are described by the empirical functional forms:

$$
\begin{gathered}
J_{i}=A_{i} \exp \left(\frac{B_{i}}{\ln ^{2} S_{i}}\right) \\
G_{i}=k_{g i}\left(S_{i}-1\right)^{\alpha_{i}}
\end{gathered}
$$

211 where $S$ is the supersaturation ratio $\left(c / c_{s a t}\right)$, and $A, B, k_{g}$ and $\alpha$ are material dependent kinetic/thermodynamic parameters. The values of these constants for the model substances used in this work are given in the results section. 


\subsubsection{Droplet heat transport model}

In addition to the mass conservation equations, an energy balance is required to describe the temperature distribution within the drying droplet. The transport of energy is similarly given by convection and conduction:

$$
\frac{\partial}{\partial t}\left(\rho C_{\mathrm{p}} T\right)=-\frac{1}{r^{2}} \frac{\partial}{\partial r}\left(r^{2} u \rho C_{\mathrm{p}} T\right)+\frac{1}{r^{2}} \frac{\partial}{\partial r}\left(r^{2} \lambda \frac{\partial T}{\partial r}\right)-\sum_{j}^{n-1} \rho_{j}^{\langle D\rangle} \Delta H_{\mathrm{crys}, j} \frac{\partial \varepsilon_{j}}{\partial t}
$$

where $C_{\mathrm{p}}$ is the overall specific heat capacity and $\lambda$ is the thermal conductivity of the suspension estimated as a weighted average (cf. supporting information) and $\Delta H_{\text {crys }}$ is the energy released by crystallisation.

This is complemented by the boundary conditions. Symmetry at the centre of the droplet:

$$
\frac{\partial T}{\partial r}=0 \quad \text { at } \quad r=0
$$

Assuming heat transfer to the droplet surface is by convection from the ambient gas, the boundary condition at the surface can be written as;

$$
-u \rho C_{\mathrm{p}} T+\lambda \frac{\partial T}{\partial r}=\frac{\dot{Q}_{\text {in }}-\dot{H}_{\text {out }}}{4 \pi r^{2}} \quad \text { at } \quad r=r_{s}
$$

The above equation at the boundary represents the balance in energy required to heat up and evaporate the droplet $\left(\dot{Q}_{\text {in }}\right)$, and the energy of the liquid evaporating from the surface $\left(\dot{H}_{\text {out }}\right)$.

\subsubsection{External heat and mass transport model}

The evaporation process is modelled using the original Abramzon and Sirignano [30] model. The evaporation phenomenon is described with some assumptions. This include negligible gas solubility in the liquid phase, no heat transfer by radiation and that mass transport occurs through a gas/liquid film at the droplet boundary layer. A mass balance for liquid/vapour across the interface yields the instantaneous evaporation rate $\dot{m}_{\mathrm{v}}$. When modified for a multicomponent system [31], $\dot{m}_{\mathrm{v}}$ is written as 
the sum of each evaporating component as:

$$
\dot{m}_{\mathrm{v}}=\sum_{i} 2 \pi \rho_{i, v} D_{i, v} R_{i} \widehat{S h}_{i} \ln \left(1+\mathrm{B}_{\mathrm{M}, \mathrm{i}}\right) \quad \text { for } \quad \mathrm{Re} \leq 400
$$

$$
\mathrm{B}_{\mathrm{M}, i}=\frac{w_{i, s}-w_{i, \infty}}{1-w_{i, s}}
$$

where $w_{i, s}$ and $w_{i, \infty}$ are the vapour mass fractions of the evaporating component at the surface and in the bulk gas respectively.

It is also possible to write a balance for the rate of heat transferred to the droplet, where the heat transferred is used to heat up the droplet and evaporate the solvent. This is written as:

$$
\dot{Q}_{\text {in }}=\dot{m}_{v}\left(\frac{C_{p v}\left(T_{\infty}-T_{s}\right)}{\mathrm{B}_{\mathrm{T} i}}-\Delta h_{f g}\left(T_{s}\right)\right)
$$

where $\mathrm{B}_{\mathrm{T}}$ is the heat transfer number given by: [30].

$$
\mathrm{B}_{\mathrm{T} i}=\left(1+\mathrm{B}_{\mathrm{T} i}\right)^{\psi_{i}}-1
$$


and $\psi$ in the above expression is:

$$
\psi=\frac{C_{p v}}{C_{p g}}\left(\frac{\widehat{\mathrm{Sh}}}{\widehat{\mathrm{Nu}}}\right) \frac{1}{\mathrm{Le}}
$$

where Le is the dimensionless Lewis number $\left(\mathrm{Le}=\lambda_{g} /\left(\rho_{g} D_{g} C_{p g}\right)\right.$.

\subsubsection{Phase Behaviour}

Evaporation of a droplet is a two phase problem and hence, equations describing the two phases (liquid and gas) must be solved. In addition, one needs to describe the thermodynamic equilibrium between the gas and liquid phases at the interface. The description of the liquid and gas phases have been described in Section 3.1.1 and Section 3.1.4 respectively. Since the presence of additional components within a liquid mixture can influence the vapour pressure of the liquid at the droplet surface and hence the degree of solvent flux during evaporation, it is also necessary to describe the thermodynamics at the liquid-vapour interface [29]. The description of thermodynamic equilibrium is often well represented by the modified Raoult's law $P_{i}=x_{i} \gamma_{i} P_{i, s a t}$. Here, the effect of liquid phase non-ideality in a mixture is accounted for using the activity coefficient $\gamma_{i}$. These can be described using a variety of models. In complex systems such as organic solutions, group contribution methods such as UNIFAC provide a useful and often reliable way of predicting the non-ideal liquid behaviour. For this work, pure component physical properties and correlations are obtained from a range of sources (values are tabulated in Table 2) $[34,35]$. For mixtures, composition dependent correlations are used as detailed in the supporting documentation.

\subsection{Shell formation}

Although the model described in Section 3.1 is only valid prior to shell formation, the concept and equations are similarly applicable during and after shell formation. The shell is deemed to form at a critical point, where the solid particles are unable to move sufficiently relative to each other. This critical point is affected by the properties of the particles forming the shell. Eq. (17) therefore relates the critical solid fraction $\varepsilon_{c r i t}$ for a stable packing as a function of the width of the PSD (using the mass weighted geometric standard deviation $\sigma_{g}$ of the PSD). The original version of this equation [36] fitted for particles with sphericity of 0.81 and 0.86 is extrapolated for the needle like crystals of the model 
compound used in this work (D-mannitol). An aspect ratio of 8 is selected to describe the needle-like crystals [37]. This gives a sphericity of 0.69 [38] (assuming the needle-like shapes are well described as cylindrical rods) - c.f. supporting information).

$$
\varepsilon_{\text {crit }}=0.7694-0.524 / \sigma_{g}
$$

This critical solid fraction is always reached first at the surface of the droplet, because supersaturation is at its maximum there, which causes increased particle formation and growth and therefore a higher solid fraction at the surface. The formation of a shell (somewhere between point $\mathrm{C}$ and $\mathrm{D}$ in Figure 1) starts when the simulated solid fraction at the surface reaches the critical solid fraction given by Eq. (17).

\section{Numerical Implementation}

To solve the moving boundary problem, a coordinate transformation is applied to render the boundary positions fixed. This way, the wealth of methods available in literature for solving fixed boundary problems can be applied. The radial coordinate of the droplet is transformed through normalisation into the new spatial coordinate, $\tilde{r} \in[01]$ [21]. The transformation reads:

$$
\tilde{r}=\frac{r}{r_{s}(t)}
$$

The following partial differential relations can be derived [21].

$$
\frac{\partial}{\partial r}=\frac{\partial \tilde{r}}{\partial r} \frac{\partial}{\partial \tilde{r}}=\frac{1}{r_{s}(t)} \frac{\partial}{\partial \tilde{r}}
$$

$$
\frac{\partial^{2}}{\partial r^{2}}=\left(\frac{\partial \tilde{r}}{\partial r}\right)^{2} \frac{\partial^{2}}{\partial \tilde{r}^{2}}=\frac{1}{r_{s}(t)^{2}} \frac{\partial^{2}}{\partial \tilde{r}^{2}}
$$

$$
\left.\frac{\partial}{\partial t}\right|_{r}=\frac{\partial \tilde{r}}{\partial t} \frac{\partial}{\partial \tilde{r}}+\left.\frac{\partial}{\partial t}\right|_{\tilde{r}}=-\frac{\tilde{r}}{r_{s}(t)} \frac{\mathrm{d} r_{s}}{\mathrm{~d} t} \frac{\partial}{\partial \tilde{r}}+\left.\frac{\partial}{\partial t}\right|_{\tilde{r}}
$$

As seen in Eq. (21), the partial differential equation in the newly transformed coordinate includes a virtual flux term that incorporates a velocity due to shrinkage. The liquid phase, solid phase and energy equations are solved using the finite volume scheme [39]. This converts the partial differential 
equations into ordinary differential equations (ODEs) that are coupled with the gas phase equation. The ODEs are solved numerically using a high resolution scheme, where upwind numerical flux functions are evaluated using the method detailed by Koren [40]. Although the order of accuracy can vary depending on the profiles simulated, the scheme is numerically stable as proven by Koren [40]. Very steep gradients lead to first order accuracy $O(h)$ (where $h$ is the step size in the grid), while smooth regions can have up to third order accurate solutions $O\left(h^{3}\right)$. This means that the error scales with the width of the discretization step in the particle size $(L)$ and radial coordinate $(r)$ with the mentioned orders. Since the physics of droplet drying involves phenomena that introduce sharp gradients (caused by the shrinkage in $r$ and the nucleation in the $L$ coordinate) and phenomena that smooth out gradients (diffusion), the actual order of accuracy of the solution to the system of equations is between first order $O(h)$ and third order $(O) h^{3}$. For the simulations discussed here, a semi discrete scheme is employed, where the number of grids is selected based on the convergence behaviour of the mixture simulated in order to yield good accuracy. Note that depending of the system simulated, this sometimes necessitates the use of a non-uniform grid in space. A grid size of 100 is used in the simulations shown here. Accuracy and convergence studies (see supporting documentation) show that the solution obtained in this work is near $(O) h^{2}$. In the time domain, the system of ODEs is solved using established ODE solvers; in this case Matlab's ODE45 [41], with absolute and relative error tolerance levels of $10^{-8}$.

\section{Experimental study}

\subsection{Materials}

PVP (K-30) and D-mannitol at purity $>99 \%$ were obtained from Sigma Aldrich and Alfa Aesar respectively. Ultra pure water with resistivity of $19 \mathrm{M} \Omega . \mathrm{cm}$ was obtained from a Direct-Q3UV purification system from Merck and was used for the preparation of all aqueous solutions.

\subsection{Experimental Setup}

A single droplet drying setup similar to that first described by Yarin et al. [14] is used to emulate the drying conditions. Here, a conditioned gas is introduced with controlled gas flow rate, temperature and 


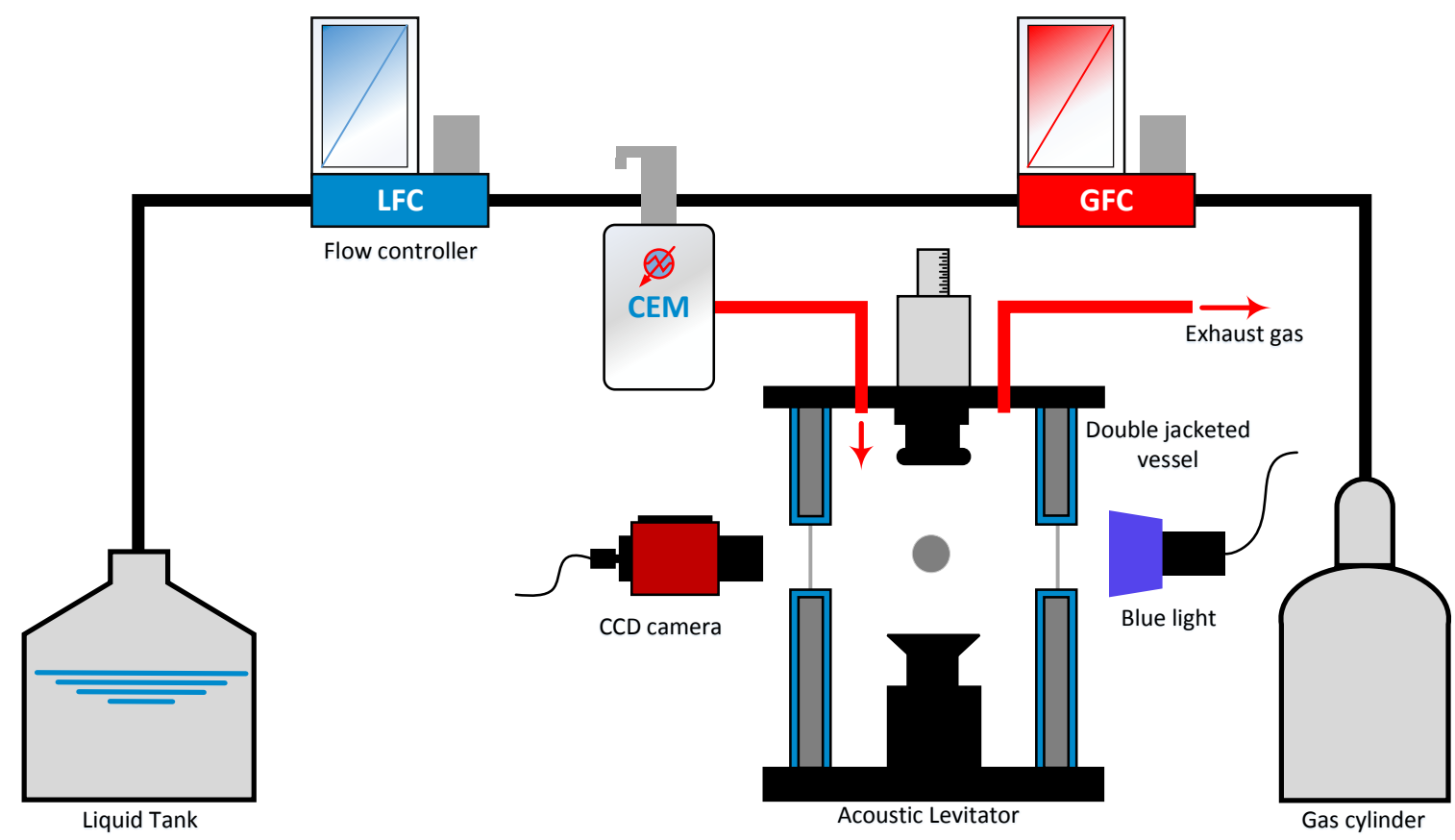

Figure 4: Scheme of the experimental apparatus used for single droplet drying. Briefly, it consists of a nitrogen gas cylinder, a liquid tank, liquid and gas flow controllers, a controlled evaporator mixer (CEM), temperature controlled chamber and an acoustic levitator which enables contactless droplet suspension. A light source and camera are used to observe the shrinking droplet. 


\subsection{Data processing}

As evaporation of the droplet progresses in the chamber, the time evolution of the droplet diameter is monitored by analysing the projected area of the droplet obtained by optical imaging. The optical images obtained from the experiments are analysed by an automated image analysis routine using MATLAB. From this, droplet/particle properties such as projected area, axis lengths, centroid etc. are extracted. To account for droplet shape deformation caused by acoustic effects, a volume equivalent spherical diameter (estimated from the projected major and minor axis length of images) is extracted and used to describe the droplet/particle size. To guarantee compatibility of model and experimental results, values used to validate the model results are based on such equivalent spherical diameters and actual initial droplet diameters as obtained experimentally. Temperature and compositional changes within are not measured due to the lack of accurate spatially resolved techniques for the small droplet.

\subsection{Experimental Conditions}

To ensure the validity of experimental results, experimental drying conditions were calibrated using a temperature and humidity sensor (Thermochron DS1923 hydrochron Ibutton; $\pm 1{ }^{\circ} \mathrm{C}$ and $\pm 2 \%$ relative humidity) placed near the droplet in the levitation field. Typically, a $1.4 \mu \mathrm{L}$ droplet is levitated, giving a droplet diameter of $\approx 1.4 \mathrm{~mm}$. Although obtaining exactly the same initial droplet size is difficult due to the manual handling of the syringe, deviations from the desired initial size are typically within $\pm 5 \%$. To test the ability of the model to capture a range of drying conditions, the effect of temperature and concentration is investigated for pure component and multicomponent systems. An overview of the experimental conditions is given in Table 1. 
Table 1: Experimental conditions used for model validation - mass fractions are expressed on a solid-free basis

\begin{tabular}{|c|c|c|c|}
\hline component(s) & temperature $(\mathrm{s})[\mathrm{K}]$ & $w_{1}(\% \mathrm{w} / \mathrm{w})$ & $w_{2}(\% \mathrm{w} / \mathrm{w})$ \\
\hline water & $313,333,343$ & 100 & 0 \\
\hline \multirow[t]{3}{*}{ Mannitol-water } & 333 & 1 & 99 \\
\hline & $323,333,343,353$ & 4.5 & 95.5 \\
\hline & 333 & 7.3 & 92.7 \\
\hline \multirow[t]{3}{*}{ PVP-mannitol-water } & 333 & 4.8 & 4.5 \\
\hline & 333 & 7.8 & 4.5 \\
\hline & 333 & 15 & 4.5 \\
\hline
\end{tabular}

\section{Results and Discussion}

The solution to the model equations given in Section 3 are implemented on MATLAB for several case studies, and are validated using experimental data as discussed in Section 5. To utilise the experimental data, drying conditions (gas temperature, humidity, velocity) must directly correlate with inputs to the model. The gas flow rate measured by the CEM (Figure 4) allows the inlet velocity to the drying chamber (for a given pipe diameter) to be determined. However, it is not possible to directly relate this velocity to the gas velocity experienced by the droplet in the drying chamber, but this is required for the calculation of the Reynold's number in Eq. (11). The velocity is obtained by treating it as a decision variable in an optimisation problem. Specifically, the $\ell^{2}$-norm of the difference between the simulated and experimental droplet diameter profile against time is minimised for the case of evaporating pure water at $\mathrm{T}=333 \mathrm{~K}$ and $\mathrm{RH}=0 \%$. This results in a gas velocity of $u_{g}=0.095 \mathrm{~m} \mathrm{~s}^{-1}$ at a gas flow rate of $1 \mathrm{~L} \mathrm{~min}-1$. Since this is a non-changing parameter for a given inlet flow rate, the value must be consistent with other systems evaporating under the same gas conditions. This was confirmed using the experimental data of several model systems (Table 1) under the same drying conditions where the model was used predictively. 


\begin{tabular}{llll}
\hline parameter & value & parameter & value \\
\hline$k_{v}$ & 0.02 & $C_{p \text {,water }}$ & $4184 \mathrm{~J} \mathrm{~kg}^{-1} \mathrm{~K}^{-1}$ \\
A & $1 \times 10^{14} \mathrm{~m}^{-3} \mathrm{~s}^{-1}$ & $C_{p \text {, mannitol }}$ & $1312 \mathrm{~J} \mathrm{~kg}^{-1} \mathrm{~K}^{-1}$ \\
B & -3 & $C_{p, \text { PVP }}$ & $2300 \mathrm{~J} \mathrm{~kg}^{-1} \mathrm{~K}^{-1}$ \\
$\rho_{\text {mannitol }}$ & $1520 \mathrm{~kg} \mathrm{~m}^{-3}$ & $\mathrm{RH}$ & $0 \%$ \\
$\rho_{\mathrm{PVP}}$ & $1300 \mathrm{~kg} \mathrm{~m}^{-3}$ & $u_{g}$ & $0.095 \mathrm{~ms}^{-1}$ \\
$\rho_{\text {water }}$ & $1000 \mathrm{~kg} \mathrm{~m}^{-3}$ & $h_{c}$ & $10 \mathrm{~W} \mathrm{~m}^{-2} \mathrm{~K}^{-1}$ \\
$\lambda_{\text {mannitol }}$ & $0.5 \mathrm{~W} \mathrm{~m}^{-1} \mathrm{~K}^{-1}$ & $\Delta H_{\text {crys }}$ & $-3 \times 10^{5} \mathrm{~J} \mathrm{~kg}^{-1}$ \\
$\lambda_{\mathrm{PVP}}$ & $0.3 \mathrm{~W} \mathrm{~m}^{-1} \mathrm{~K}^{-1}$ & $k_{g}$ & $2 \times 10^{-6} \mathrm{~m} \mathrm{~s}^{-1}$ \\
$\lambda_{\text {water }}$ & $0.1 \mathrm{~W} \mathrm{~m}^{-1} \mathrm{~K}^{-1}$ & $\alpha$ & 1.2 \\
\hline a All the values shown here are assumed constant in the liquid phase. Temperature dependent proper- \\
ties used in the model are given as part of the supplementary information.
\end{tabular}

\subsection{Non-particle forming systems}

\subsubsection{Evaporation of Pure component droplets}

Firstly, the behaviour of the model is examined using the droplet drying history of pure component systems. The rate at which the radius of the droplet is decreasing is given by:

$$
\frac{\mathrm{d} r_{s}}{\mathrm{~d} t}=-\frac{\dot{m}_{\mathrm{v}}}{4 \pi r_{s}^{2} \rho^{\langle C\rangle}}
$$

Godsave [42] and Spalding [43] showed that when the temperature of the droplet reaches the wet bulb temperature, usually after a rapid initial heating period, quasi-steady equilibrium is achieved and the well established $d^{2}$ law (Eq. (23)) can be derived, assuming a gas-phase model i.e., negligible liquid phase heat and mass transfer (see supporting information for the derivation).

$$
\frac{\mathrm{d}\left(d^{2}\right)}{\mathrm{d} t}=k t
$$




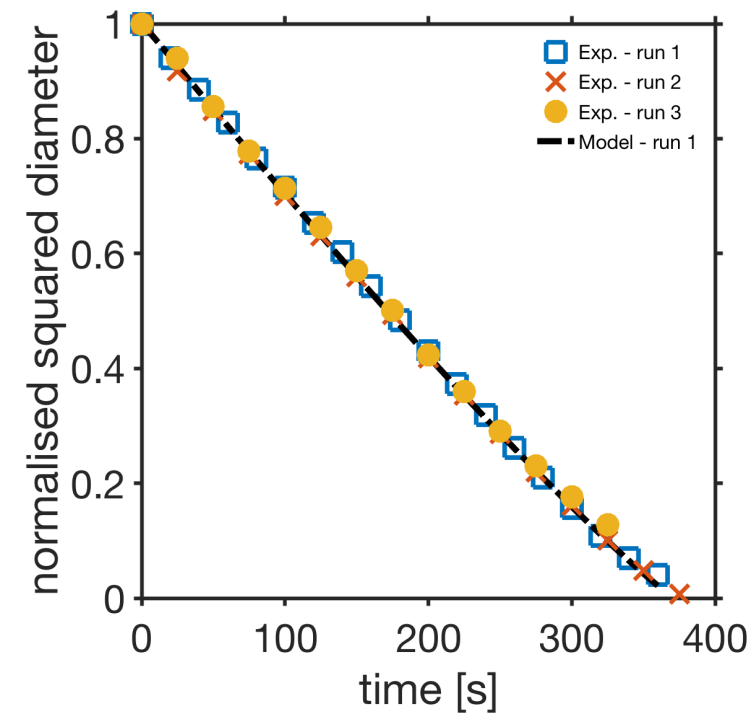

(a)

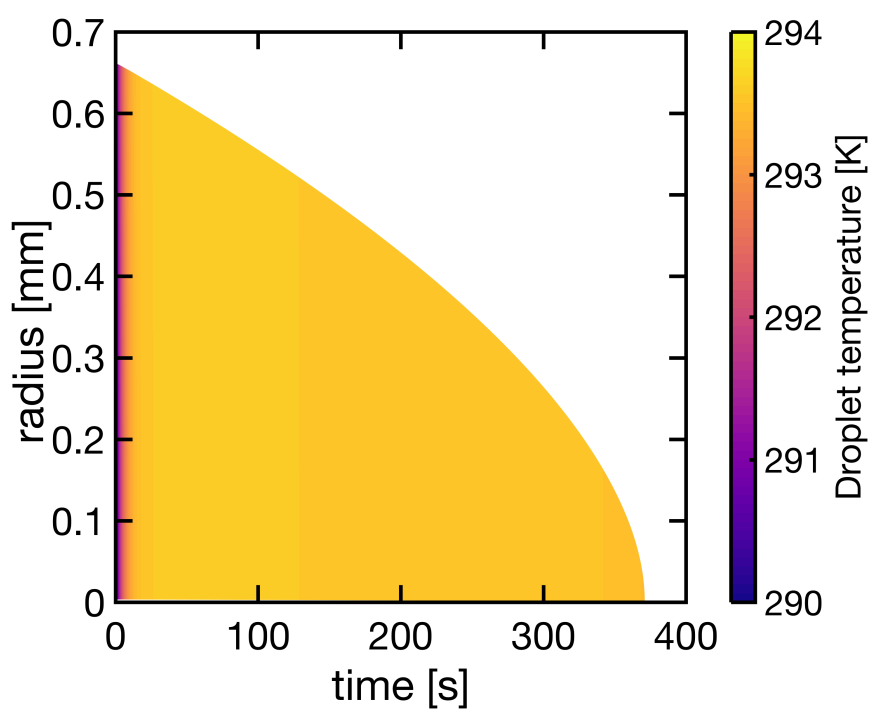

(b)

Figure 5: Evaporation of pure water at $333 \mathrm{~K}$ and $0 \%$ relative gas saturation. Data points are recorded every second, but for display purposes fewer are shown. The value of some dimensionless numbers and parameters at the beginning of drying $(290 \mathrm{~K})$ and with a starting droplet diameter of $1.5 \mathrm{~mm}$ are: $\mathrm{Re}=7.9, \mathrm{Sc}=0.67, \mathrm{Sh}=3.40, \mathrm{Sh}=3.39, \mathrm{Le}=0.92, \operatorname{Pr}=0.73$, $\mathrm{B}_{\mathrm{M}}=0.013, \mathrm{~B}_{\mathrm{T}}=0.028$. The time evolving profile of the Reynold and Sherwood numbers are further illustrated in Figure $\mathrm{S} 1$ of the supporting information. The value of the Biot number $\left(\mathrm{Bi}=2 h_{c} r_{s} / \lambda\right)$ obtained for a maximum heat transfer coefficient $h_{c}$ of $10 \mathrm{~W} \mathrm{~m}^{-2} \mathrm{~K}^{-1}$ is 0.03 .

where $k$ is the evaporation constant. The above equation states that a plot of the droplet surface area against time is linear for a pure component system. By normalising the droplet surface area at every time with the initial surface area, it is indeed also more convenient to compare the drying history of different droplets as experimentally observed. Figure 5 shows the evolution of the droplet diameter and simulated temperature distribution for pure water. As expected for the case of pure water, the model

the drying process). This can be rationalised by the relative motion between the droplet and the gas phase. The Reynolds number is continuously decreasing during drying (cf. Figure S1 in the supporting information). This change is in fact not accounted for in the derivation of the classical $d^{2}$ law [44]. The evaporation model described here accounts for these effects. The results shown in Figure $5 \mathrm{~b}$ further illustrate the capability of the model to describe the evaporation phenomena down to very small sizes $(1 \mu \mathrm{m})$; the physical sizing limit of the camera. This is based on the observation that the model and experimental data are still in good agreement at these small sizes and therefore, this ability is in- 

drying at different temperatures. The model results similarly show excellent prediction for drying at

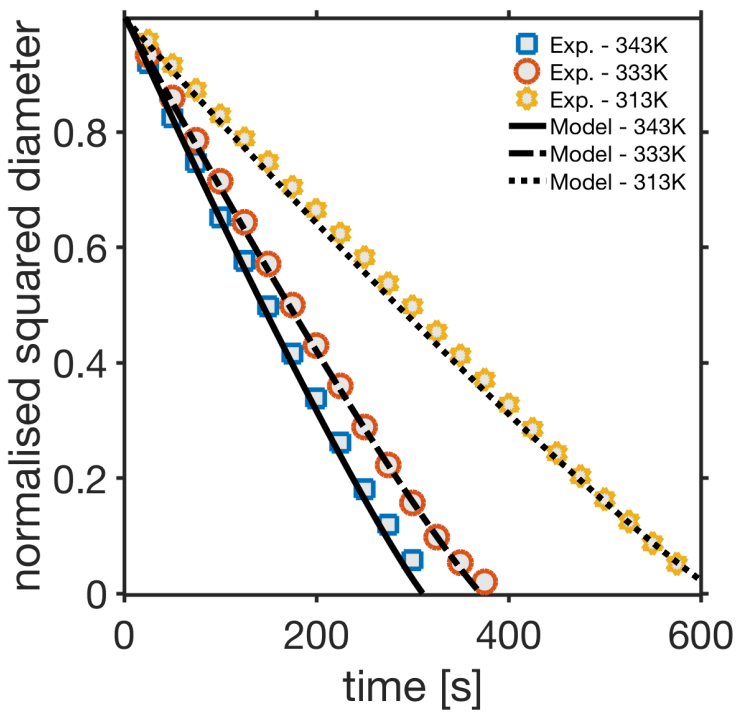

(a)

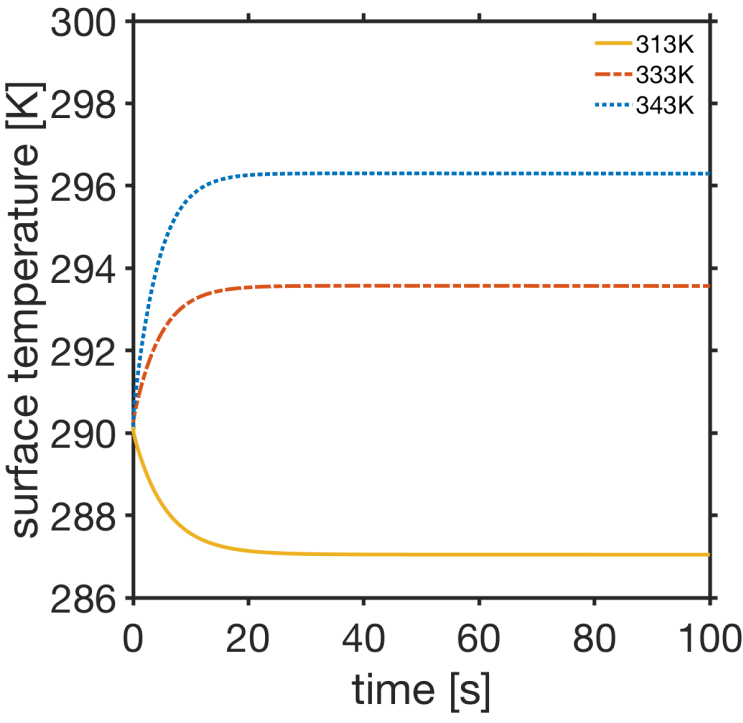

(b)

Figure 6: Effect of gas temperature on the evaporation of water at $0 \%$ relative gas saturation. a) evolution of the normalised squared droplet diameter b) evolution of the surface temperature of the droplet.

dependent from the initial size of the droplet. This is an important observation, because the initial droplet size employed in the experiments (due to physical limitations) is much larger than those typically employed within spray drying operations. The temperature distribution in Figure $5 \mathrm{~b}$ shows that the temperature gradient within the droplet is negligible, which is a consequence of the small droplet size and the high thermal conductivity of water. This can be further rationalised by the low Biot number calculated $(\mathrm{Bi}=0.03$; see the caption of Figure 5 for further characteristic dimensionless numbers). Although the above results for water are based on values of optimised velocity experienced by the droplet, by drying pure water droplets at different temperatures under the same drying gas condition, one can determine the general applicability of the model. Figure 6 shows drying results obtained for

different temperatures. As expected, the evaporation rate increases with an increase in temperature due to a larger temperature driving force at constant relative saturation. In Figure 6, this is reflected by the shorter drying time and the faster decrease in droplet diameter. As mentioned previously, the measurement of droplet temperature is difficult experimentally with the resolution of applicable methods being quite limited. Hence, surface temperatures obtained from the model here are compared to experimentally observed wet bulb temperatures available from psychrometric charts $[45,46]$, see Table 3 . The predicted surface temperatures are also in good agreement with the experimentally observed wet bulb 
temperatures reported in literature. The validity of the model is further substantiated in the supporting information where data for other pure components (methanol, ethanol, isopropanol) as well as binary mixtures of these solvents are shown.

Table 3: Comparison of experimental wet bulb temperatures and predicted surface temperatures ${ }^{\mathrm{a}}$

\begin{tabular}{llll}
\hline component & gas temperature $[\mathrm{K}]$ & exp. wet bulb temperature $[\mathrm{K}]$ & model surface temperature $[\mathrm{K}]$ \\
\hline \multirow{2}{*}{ water } & 313 & 287 & 287.0 \\
water & 333 & 293 & 293.6 \\
\multirow{2}{*}{ water } & 343 & 295 & 296.3 \\
\hline
\end{tabular}

${ }^{\text {a }}$ All simulations and experiments are at $0 \%$ relative saturation of the gas phase $\left(\mathrm{N}_{2}\right)$.

\subsection{Particle forming systems}

In order to validate the model for systems forming particles within the droplet, a homogeneous aqueous solution of mannitol and a ternary solution containing mannitol and PVP is considered. The activity of components in all solutions are determined using the UNIFAC group contribution method as discussed in Section 3.1.5. Details of the UNIFAC model are provided in the supporting documentation.

\subsubsection{D-Mannitol in water}

In the drying of a homogeneous solution containing mannitol and water, the initial droplet is a clear undersaturated solution (starting concentration, $7.3 \% \mathrm{w} / \mathrm{w}$ solution), with the solute and temperature distribution assumed to be uniform initially within the droplet. The temperature dependence of the binary diffusion coefficient in solution is modelled using the Stokes-Einstein equation [29]. Due to the formation of solid particles within the droplet, an effective diffusivity must be considered to account for the effect of the evolving solid particles on drying. The effective diffusivity $D_{\text {eff }}$, which is a combination of both surface diffusion and pore diffusion is accounted for using the Clausius-Massotti model [47]. Since D-mannitol adsorbs water very weakly [48], one can assume that surface diffusion is negligible. 
This simplifies the Clausius-Massotti model to Eq. (24):

$$
D_{\text {eff }}=\frac{2 D(1-2 \varepsilon)}{2+\varepsilon}
$$

where $D$ is the pore diffusion coefficient (derived from Stokes-Einstein equation). This diffusion coefficient is used in all relevant conservation equations. The nucleation and growth kinetics of D-mannitol are based on the empirical functional forms Eq. (7) and Eq. (8). The kinetic constants used for the simulation here is shown in Table 2 and are that obtained from Cornel et al. [49], except the value of $A$ which represents the maximum nucleation rate. As a result of the complexity of nucleation processes, the value of the maximum nucleation rate can vary between similar systems and process scales. In the present model application, this parameter is obtained by monitoring the time at which shell formation is observed. This analysis gives a reasonable nucleation rate of $A=1 \times 10^{14} \mathrm{~m}^{-3} \mathrm{~s}^{-1}$ [49] ${ }^{1}$. The results obtained from the simulation for the droplet diameter profile is shown in Figure 7 and an excellent prediction is made up to the critical point. The concentration profile of mannitol illustrates that as the solution droplet dries, a concentration gradient is built up within the droplet over time with the highest concentration of solute at the droplet surface (Figure 8a). The concentration increases due to a reduction in droplet size as a result of evaporation. After the saturation point of the solute in the solvent is reached, continuous evaporation and droplet shrinkage causes the concentration to exceed the saturation concentration, providing a driving force in terms of supersaturation for crystallisation. The solid fraction (Figure 8b) increases very quickly towards the end of drying due to the exponential increase in nucleation rate (Eq. (7)) with supersaturation. This is complemented by a much larger number of crystals growing towards the end of the first drying stage. Shell formation (critical point) is predicted at a solid fraction of 0.32 , at $\approx 266 \mathrm{~s}$ and final droplet diameter of $0.8 \mathrm{~mm}$, which matches the experimental observation well (Figure 7; $270 \mathrm{~s}$ experimentally). When drying is allowed to continue until a solid fraction of 0.7 , shell formation occurs at $271 \mathrm{~s}$, with a final droplet diameter of 0.76 . This shows that the effect of PSD on the critical point may not be significant for the model system considered here. At the critical point, the shell can lock due to the presence of electrostatic interaction between particles

\footnotetext{
${ }^{1}$ While this value is different from the one reported in Cornel et al. [49] for the $\alpha$ polymorph, it is important to remember that the original authors did not quantitatively estimate these values from experimental data, but merely have used it to reconstruct the qualitative behaviours seen in their data.
} 


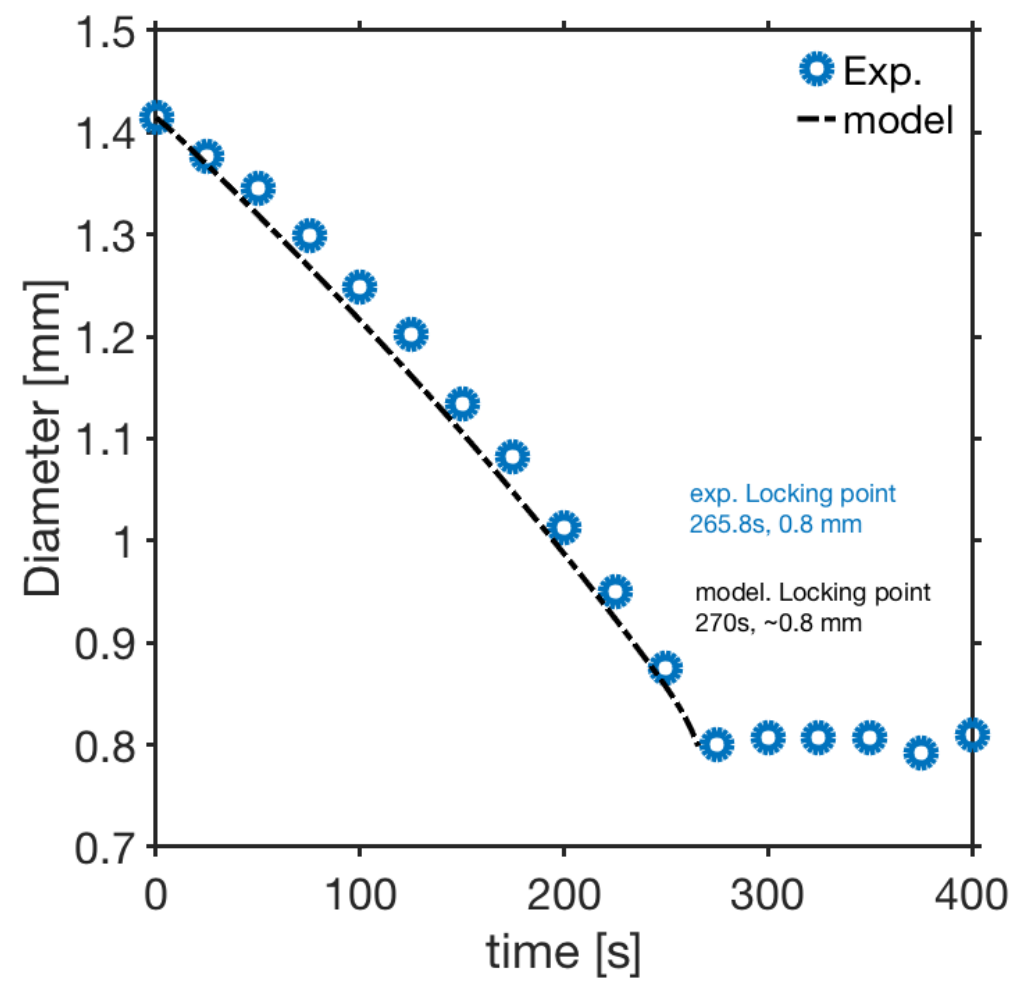

Figure 7: Model and experimental results for the drying history of a droplet containing an aqueous solution of D-mannitol $(7.3 \% \mathrm{w} / \mathrm{w} \mathrm{D}$-mannitol) at $333 \mathrm{~K}$ and $0 \%$ relative gas saturation.

at the particle-dense surface. However, this must be balanced by the ability of the shell to withstand surrounding pressures (external and capillary pressures) $[20,50]$. When a strong shell is not formed instantaneously, there may exist a droplet-particle transition regime (shell thickening regime) where the pressures exerted on the forming shell causes a cycle of shell buckling events until the shell is capable of withstanding the stress forces acting on the droplet/particle surface [50]. As such, the droplet may still continue to shrink, albeit more slowly due to the high resistance to evaporation at the particle dense surface. Nevertheless, the results shown here for this model system do not show this behaviour (as seen from the constant particle diameter after the critical point; cf. Figure 7). This highlights that the model devised here is applicable to this model system. As expected from the temperature profile of the droplet (Figure 9), the droplet temperature rises to the wet bulb temperature of the solution. Since the concentration of the solution is not constant, the wet bulb temperature varies slightly over time and will depend on the evolving physical properties of the liquid mixture. Near the critical moisture content, the temperature begins to rise to the gas temperature (cf. Figure 1) when drying below the boiling point. Figure 10 shows an example particle size distribution (PSD) during drying. This figure 


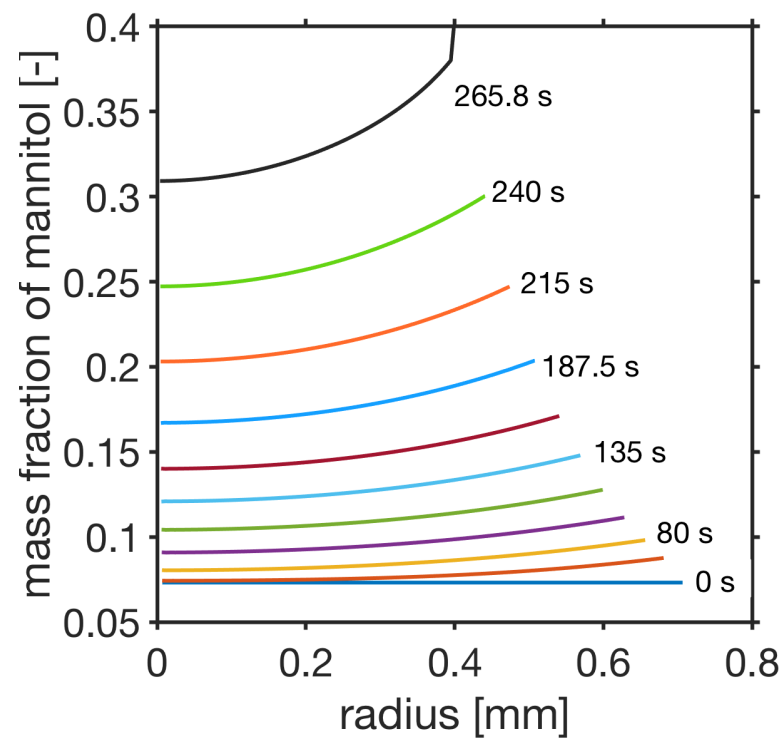

(a)

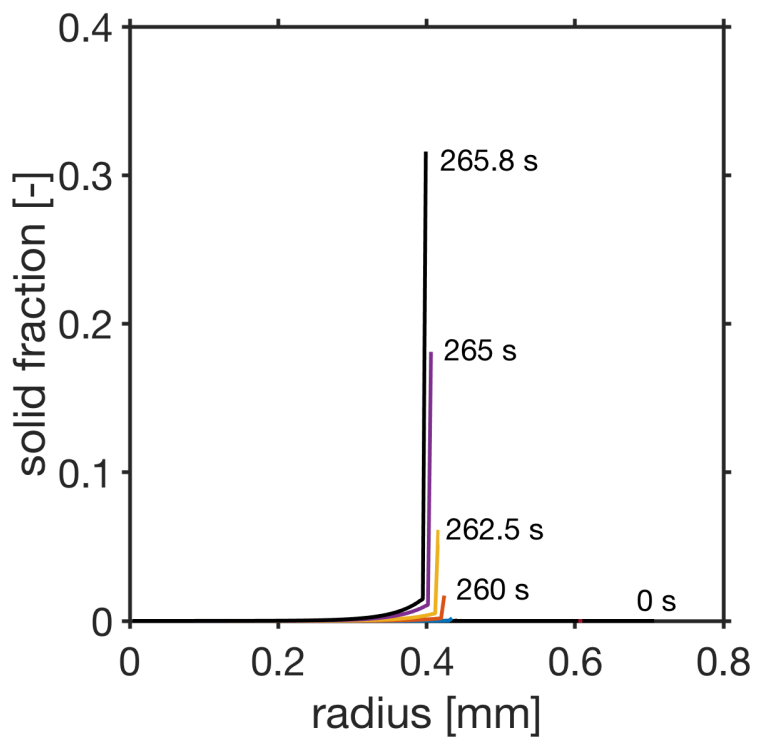

(b)

Figure 8: simulated results for the a) concentration and b) solid fraction profile of a droplet containing an aqueous solution of D-mannitol (7.3\% w/w D-mannitol) drying at $333 \mathrm{~K}$ and $0 \%$ relative gas saturation.

represents the PSD present at all radial coordinate points at $240 \mathrm{~s}$. Therefore, a horizontal line in this figure represents the PSD at a specific radial location, with the value of the PSD shown with a colour scale (see colour bar on the right; note the logarithmic scale). The PSD at the liquid/gas interface ( $\mathrm{r}$ $\approx 0.42 \mathrm{~mm}$ ) shows both the largest particles and the highest number of particles. This is due to the supersaturation at the interface being the highest throughout the drying process, which results in high nucleation and growth rates (cf. Eq. (7) and Eq. (8)). To allow a more quantitative evaluation a series of time evolving profiles of reduced properties of the PSD will be shown. Specifically, the integrals of the PSD along the $L$ and $r$ direction is introduced:

$$
\begin{aligned}
& N_{i, \mathrm{~L}}(t, r)=\int_{0}^{\infty} N_{i}(t, r, L) \mathrm{d} L \\
& N_{i, \mathrm{r}}(t, L)=4 \pi \int_{0}^{r_{s}} r^{2} N_{i}(t, r, L) \mathrm{d} r
\end{aligned}
$$




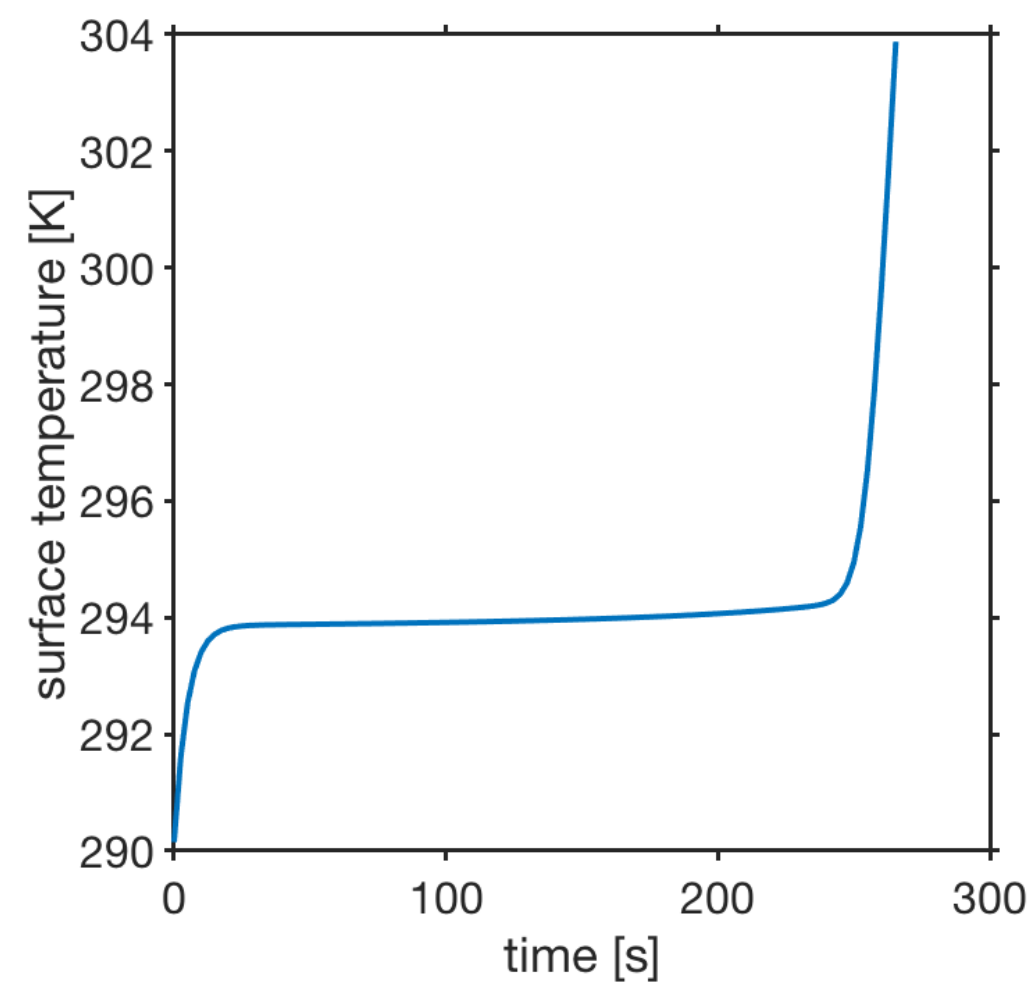

Figure 9: simulated temperature profile of $7.3 \% \mathrm{w} / \mathrm{w}$ aqueous mannitol solution droplet drying at $333 \mathrm{~K}$ and $0 \%$ relative gas saturation.

Figure 11a illustrates the time evolution of $N_{i, \mathrm{r}}$, where the total PSD within the droplet (acting as if it is well mixed) is shown. At the beginning of drying, there are no particles and as drying proceeds and the solution becomes supersaturated, nucleation occurs at small sizes and growth of crystals is observed in the droplet. It can also be observed that a smaller number of larger crystals exists within the droplet when compared to the number of smaller crystals present. This results from the fact that the supersaturation achieved within the droplet becomes much higher as the droplet becomes smaller. Just prior to shell formation (260 - 265s in Figure 8b), the supersaturation is consumed significantly that the number of nuclei formed within the droplet begins to decrease again (seen as a dip of $N_{i, \mathrm{r}}$ at small sizes). The results also show that particles up to $100 \mu \mathrm{m}$ can be produced within the droplet. An exemplary scanning electron microscopy (SEM) image of the final particle obtained (Figure 12) shows that this is indeed very possible. Figure $11 \mathrm{~b}$ shows the evolution of $N_{i, \mathrm{~L}}(t, L)$, with the concentration of particles highest at the droplet surface. Due to advection and diffusion, a significant amount of particles are also formed at the centre of the droplet. 


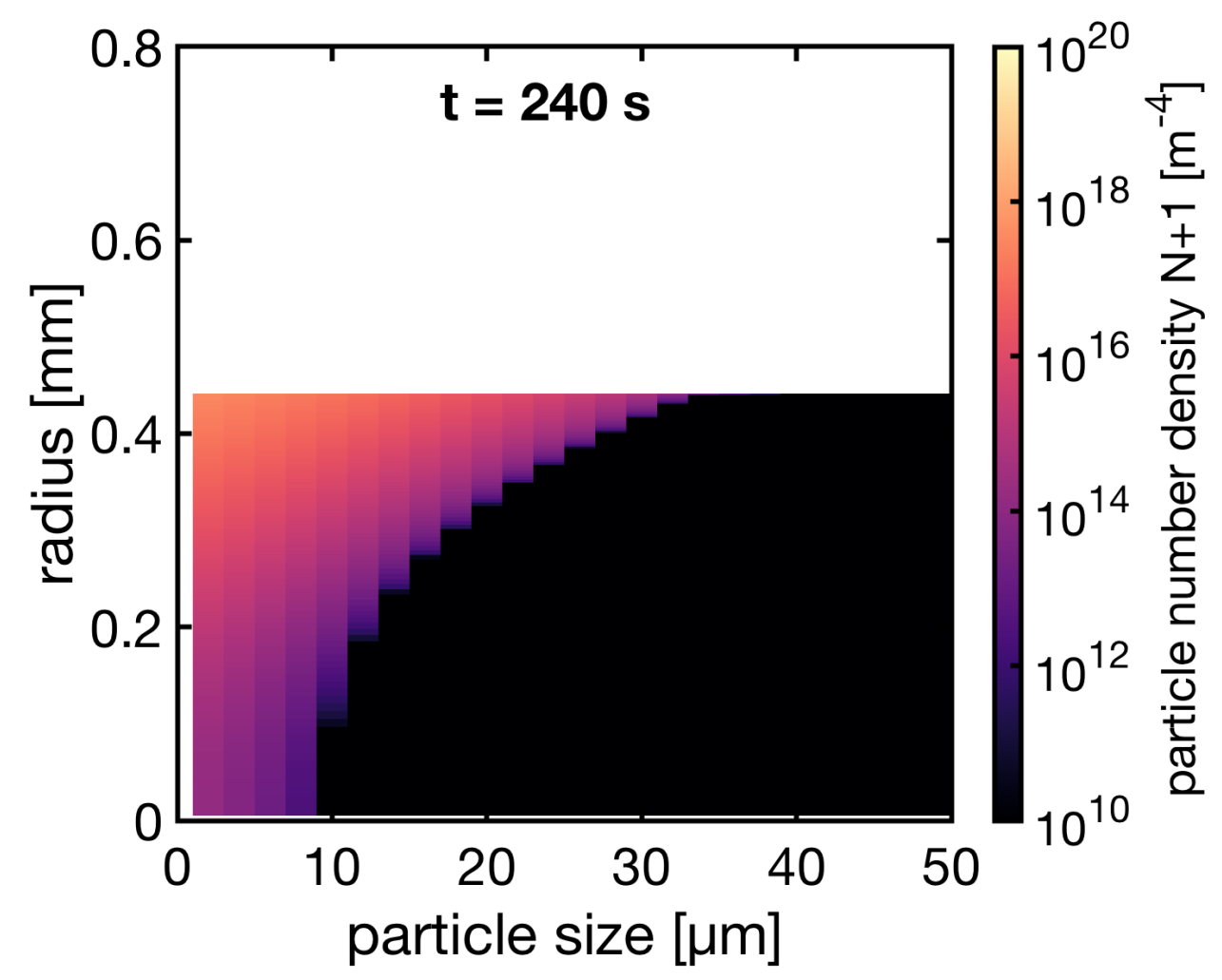

Figure 10: Simulated particle size distribution (PSD) of mannitol at $240 \mathrm{~s}$.

In order to further test the predictability of the model, droplets drying at different gas temperatures and starting concentrations are investigated. The predicted drying profile of the droplets is shown in Figure 13. These profiles indicate an excellent predictability of the drying history for the range of conditions considered. The key results obtained are summarised in Table 4. As the concentration of mannitol increases from $0.9 \% \mathrm{w} / \mathrm{w}$ to $11.2 \% \mathrm{w} / \mathrm{w}$, the time of shell formation reduces owing to the faster and higher solute enrichment at the droplet boundary. This leads to faster particle formation kinetics and hence, droplets with high initial solute concentration will produce particles with larger sizes, as opposed to droplets with a lower starting concentration. In the case of drying at different temperatures, a good prediction is also made ${ }^{2}$, with the time of shell formation seen to decrease due to the higher evaporation rates. However, a significant trend is not observed for the final particle diameter. This shows that the competition between the convective process and particle formation may be significant

\footnotetext{
${ }^{2}$ The result presented for the different temperatures here assumes that the particle formation kinetics do not change with temperature. In reality, the nucleation and growth rate of particles increases with temperature. The predicted result however still shows good comparisons with experiment.
} 


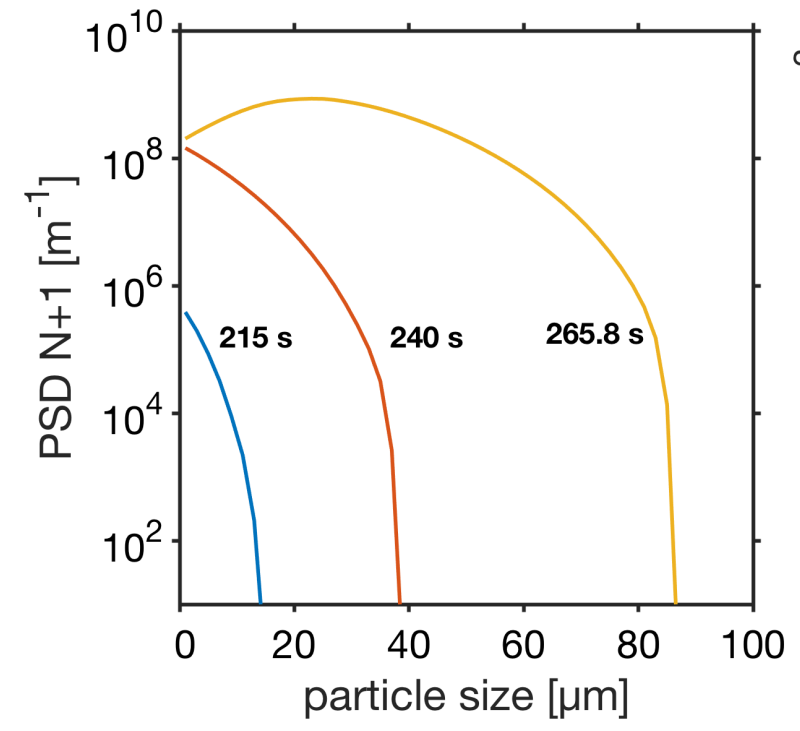

(a)

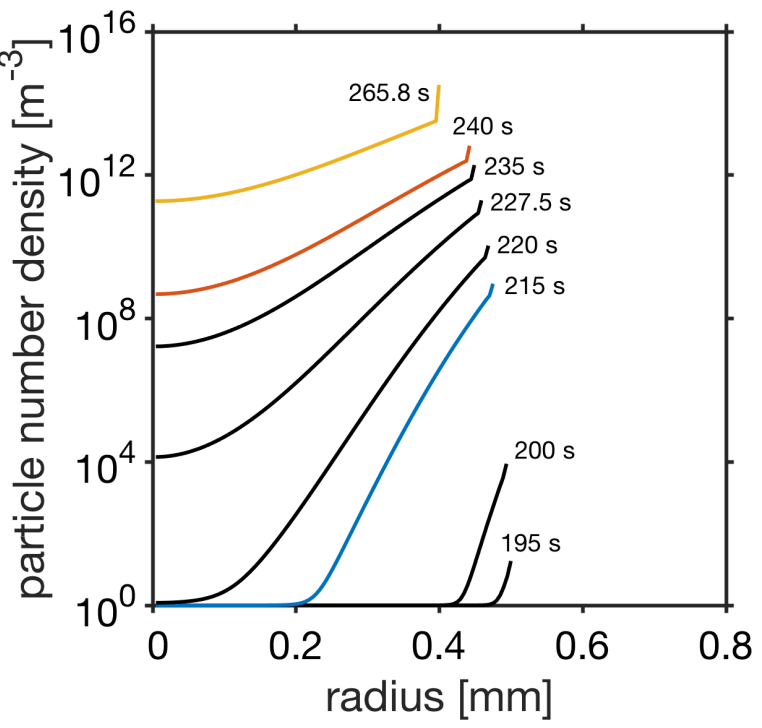

(b)

Figure 11: a) evolution of the total particle number density in the droplet, assuming a well mixed droplet b) evolution of the total particle number density at each radial location during the drying of a droplet of $7.3 \% \mathrm{w} / \mathrm{w}$ aqueous mannitol solution at $333 \mathrm{~K}$ and $0 \%$ relative gas saturation.

497 when drying particle forming formulations. Analysis of the Peclet number $\mathrm{Pe}=\frac{k}{8 D}$ shows that the Peclet number decreases, although not significantly as the starting concentration of solute is increased (Table 4). Again, no trend is particularly observed in the case of varying drying temperatures. Overall, the results indicate that the model is able to predict droplet size and the time of shell formation with good accuracy for a large range of initial concentrations and for the range of temperatures considered. 


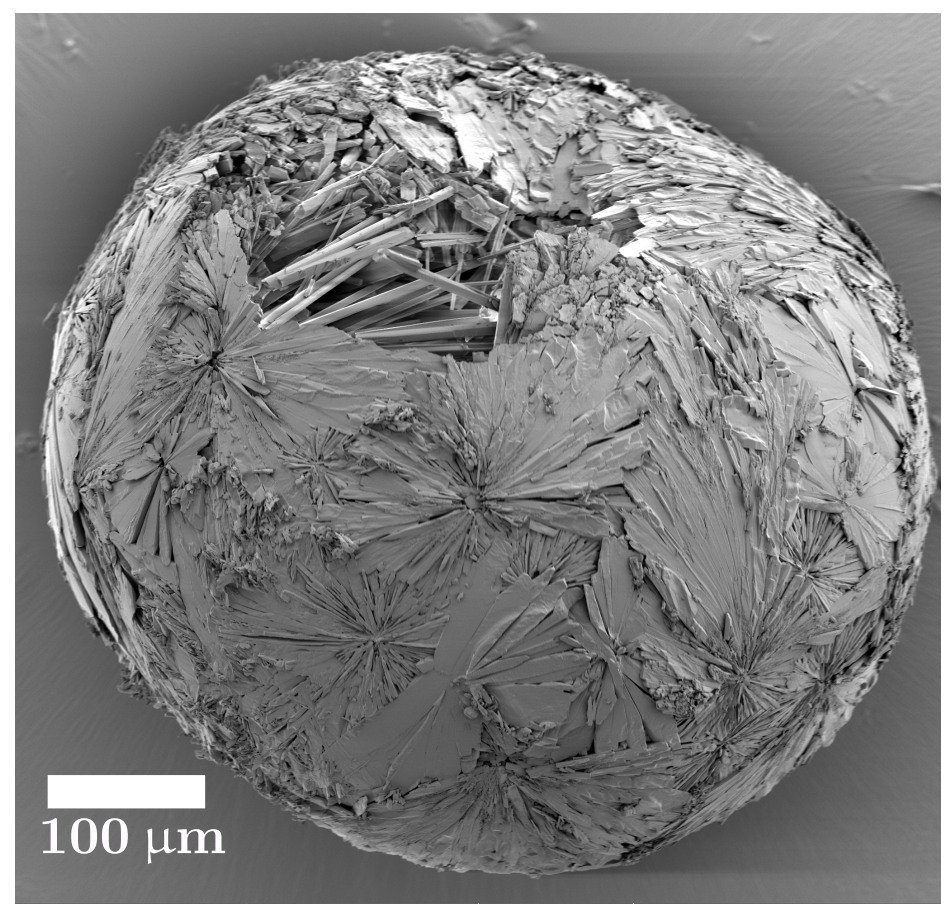

Figure 12: SEM image of a mannitol particle manufactured from a droplet with initial concentration of $4.5 \% \mathrm{w} / \mathrm{w}$ mannitol in water, and dried at $313 \mathrm{~K}$ and $0 \%$ relative saturation. The final particle structure supports the crystallization model used, and also shows that crystal sizes larger than $100 \mu \mathrm{m}$ can be obtained as illustrated in Figure 11.

Table 4: Experimental and model results for the drying of mannitol solutions for different initial droplet concentrations and temperature ${ }^{\mathrm{a}}$.

\begin{tabular}{llllllll}
\hline Initial mannitol concentration $w_{\text {initial }}(\% \mathrm{w} / \mathrm{w})$ & 0.9 & 4.5 & 4.5 & 4.5 & 4.5 & 7.3 & 11.2 \\
\hline Initial droplet diameter $[\mathrm{mm}]$ & 1.41 & 1.24 & 1.36 & 1.36 & 1.27 & 1.41 & 1.31 \\
Drying temperature [K] & 333 & 323 & 333 & 343 & 353 & 333 & 333 \\
Evaporation constant $k\left[\times 10^{-9} \mathrm{~m}^{2} \mathrm{~s}^{-1}\right]$ & 4.96 & 3.90 & 5.18 & 5.89 & 6.23 & 5.24 & 5.18 \\
Peclet number [-] & 0.95 & 0.75 & 0.94 & 0.96 & 0.94 & 0.92 & 0.87 \\
Final experimental droplet diameter [mm] & 0.37 & 0.57 & 0.63 & 0.61 & 0.63 & 0.81 & 0.86 \\
Final predicted droplet diameter $[\mathrm{mm}]$ & 0.36 & 0.59 & 0.63 & 0.62 & 0.56 & 0.78 & 0.86 \\
Experimental critical point [s] & 380 & 315 & 280 & 255 & 195 & 270 & 190 \\
Predicted critical point $[\mathrm{s}]$ & 381 & 291 & 290 & 245 & 195 & 266 & 189 \\
\hline
\end{tabular}

a All simulation and experimental data shown are obtained at $0 \%$ relative saturation of the gas phase $\left(\mathrm{N}_{2}\right)$ and a gas velocity of $0.095 \mathrm{~m} \mathrm{~s}^{-1}$. The Peclet number is calculated using an average $k$ value i.e., average of the $k$ values obtained at every time step and the diffusion coefficient is calculated using the starting droplet concentration. 


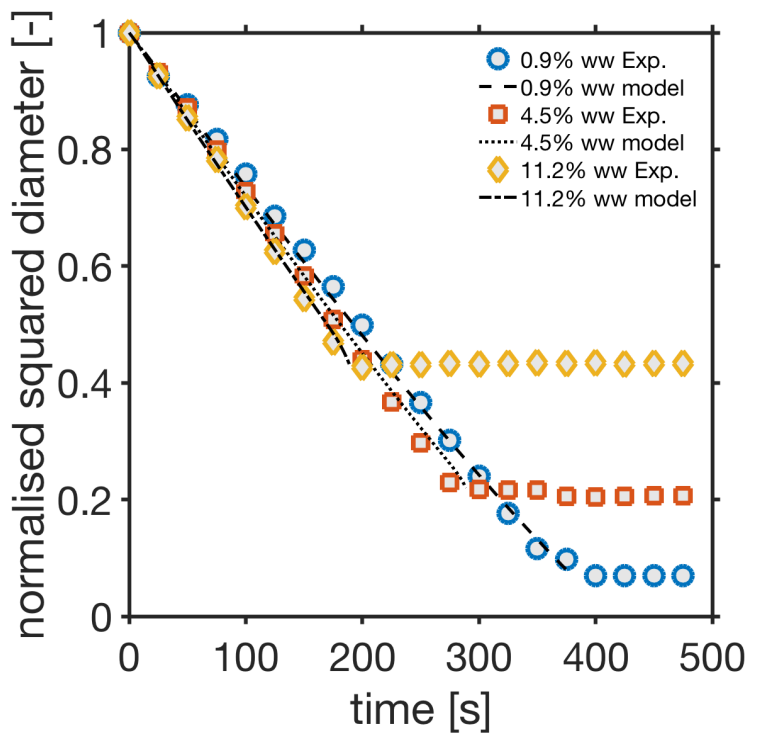

(a)

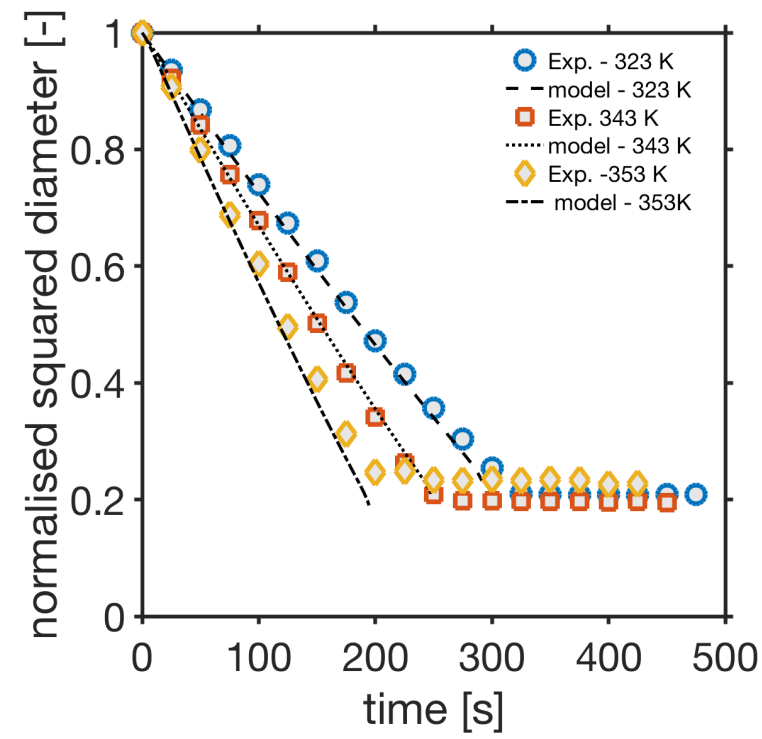

(b)

Figure 13: Droplet drying history of mannitol at a) different concentrations and b) different temperatures.

\subsection{2. $P V P$ and D-Mannitol in water}

The evaporation of a ternary system containing PVP and D-mannitol in water is now considered. Since experimental data for diffusion for multicomponent systems are often unavailable, multicomponent diffusivity must be predicted instead. Cussler [29] showed that the well established relations for Maxwell-Stefan diffusion coefficients can be related to Fick's diffusion coefficient using thermodynamic relations. In this work, such complexity is streamlined for a more simplistic, but physically reasonable approach. The diffusive fluxes of the solutes in such solute-solute-solvent systems can be treated independently (assuming the cross diffusion coefficients are small) to yield estimates of diffusivity [51]. By assuming the fluxes are relative (i.e. the total volume flux of solutes is equal to the solvent flux) and combining this with the ratio of the diffusive flux of the two solutes i.e., $s=\frac{D_{13}}{D_{23}} \frac{\frac{\partial c_{1}}{\partial t}}{\frac{\partial c_{2}}{\partial t}}$, one can relate the independent solute diffusivity to the diffusivity of the solvent to give the expressions [51];

$$
D_{1}=s \frac{\rho_{1} \rho_{2}}{\rho_{3}\left(\rho_{2} s+\rho_{1}\right)} D
$$

$$
D_{2}=\frac{\rho_{1} \rho_{2}}{\rho_{3}\left(\rho_{2} s+\rho_{1}\right)} D
$$


where the subscript 1,2,3 denote the D-mannitol, PVP and water, respectively and $D$ is the binary diffusivity of the dominant solute (depending on the starting concentrations). To describe the non-ideality of the solution, the standard UNIFAC procedure combined with a free volume parameter estimation [27] is used. The change in solubility of mannitol in the mixture is assumed to be negligible i.e., the solubility is the same as in water. This assumption may affect the degree of supersaturation reached within the droplet and hence the extent of nucleation and growth of particles at any given time. This effect is however expected to be reduced since the higher viscosity of polymer mixtures also lead to a reduction in the kinetics of particle formation. The binary diffusion coefficient of PVP in aqueous solutions is estimated from experimental data [52]. As discussed in Section 6.2.1, mannitol solidifies to form crystals. The phase behaviour of PVP can be described by its viscosity profile (Figure 14a), where the viscosity increases exponentially with concentration. This results from the cross-linking of the long-chain polymer in the solvent. At a sufficiently high concentration, the degree of cross-linking is high so that the polymer forms a stable 3-dimensional solid-like structure - a gel. During the first drying stage, the polymer will act to inhibit the kinetics of crystal formation of mannitol due to the continuously changing solution properties, specifically by altering the solution viscosity and, potentially, the interfacial tension between the liquid and solid; both of which affect the kinetics of nucleation and growth. Increasing the viscosity decreases the rate constants of nucleation and growth, $A$ and $k_{g}$ $[53,54]$. Increasing interfacial tension increases the thermodynamic parameter of the nucleation rate [53]. However, Bolten and Türk [55] showed that the surface tension of PVP/water solutions is nearly constant over a wide range of concentrations, thus the parameter $B$ is kept unchanged. $A$ and $k_{g}$ on the other hand are adjusted by multiplying them with the ratio of the viscosity of water to the polymer solution [53]. The gelation of PVP in a binary solution of PVP and water is predicted from experimental viscosity data [56], where the viscosity increases exponentially with PVP concentration. The value of the gelation fraction is taken as $50 \% \mathrm{w} / \mathrm{w}$ concentration (Figure 14a) i.e., the point where the viscosity of the solution increases sharply. The simulation of the drying history of the binary mixture of PVP and water is shown in Figure 14b and compares well with experimental results. When the drying history of the droplet for the binary solution of PVP-water (Figure 14b) and mannitol-water (Figure 8) is compared, the formation of a shell occurs at similar times. In the ternary solution of PVP-mannitol-water, defining a critical point is more complex due to the functional relationship between the solutes, crystals and the gelation property of the polymer. The critical point in such a ternary solution is neither the 


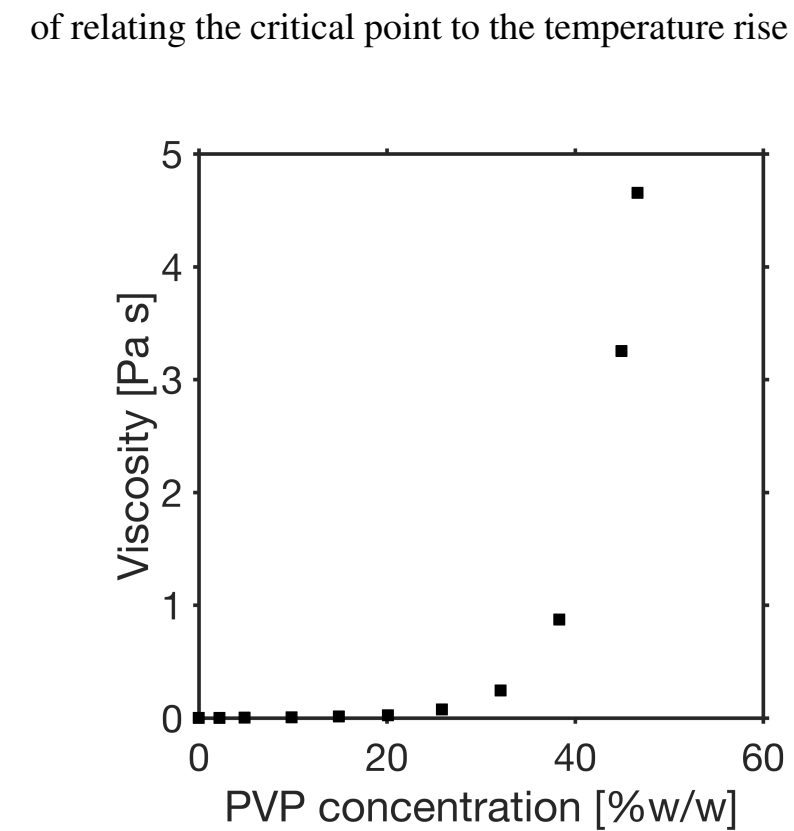

(a)

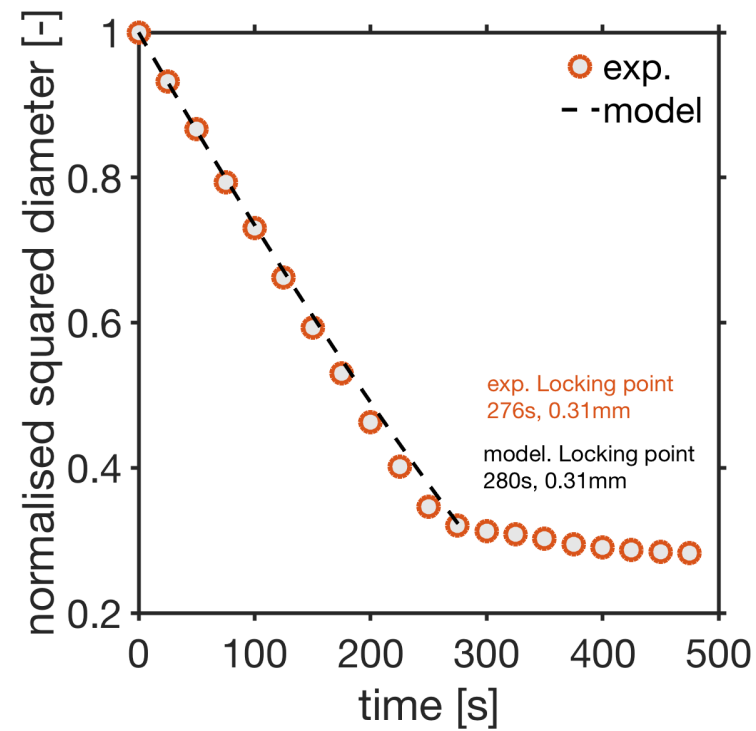

(b)

Figure 14: a) Viscosity data of PVP in aqueous solutions adapted from Ashland [56], b) drying behaviour of a droplet of aqueous solution of PVP $(6 \% \mathrm{w} / \mathrm{w})$ at $333 \mathrm{~K}$ and $0 \%$ relative gas saturation.

gelation point of the pure polymer nor the critical solid fraction of the evolving crystals. Instead, it is a value that is expected to be below the critical values of the binary solutions. The critical point can instead be empirically observed from the fact that the droplet surface temperature rises at some point due to the increased resistance to evaporation caused by the accumulation of solutes/particles at the surface (this is represented as point $\mathrm{C}$ in Figure 1). Note that in the binary case of mannitol-water (cf. Figure 9), the temperature rise is also observed near the point of shell formation. In the present application on a ternary system, a critical temperature is defined as an indication of shell formation. A value of $300 \mathrm{~K}$ ( $\approx 2.5 \%$ higher than the wet bulb temperature $(\approx 293 \mathrm{~K})$ observed during most of the first drying stage Figure 9) is used, and therefore shell formation will occur when the surface temperature of the droplet reaches the predefined critical temperature. While this value is somewhat arbitrary, the methodology of relating the critical point to the temperature rise is physically sound (cf. Section 2). Figure 15 shows

the evolution of the droplet diameter for PVP-mannitol-water solution (4.8\% w/w PVP, $4.5 \%$ w/w mannitol). The model also predicts the drying history well in this case. In this plot, a comparison of the methodology of shell formation at a critical temperature is compared to a case where the temperature is allowed to rise until it reaches the gas temperature (when drying below the boiling point). In the latter case, there is a deviation from the experimentally observed profile. This behaviour is not surprising 


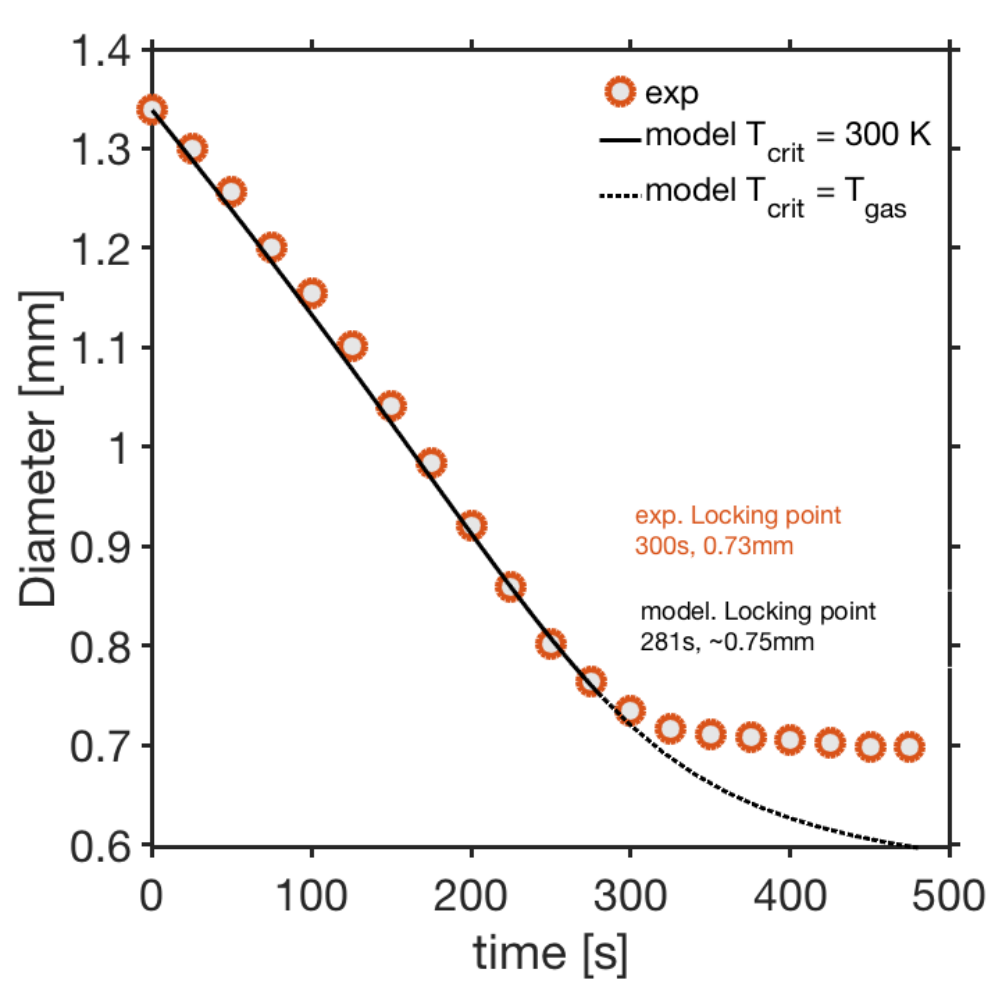

Figure 15: The drying history of a droplet containing an aqueous solution of PVP and D-mannitol 4.8\% w/w PVP, $4.5 \%$ $\mathrm{w} / \mathrm{w}$ mannitol and drying at $333 \mathrm{~K}$. The solid line (-) shows the drying profile and critical point when a critical temperature is predefined, while the dotted line (..) shows the profile when drying is allowed to continue until the surface temperature reaches the gas temperature.

since shell formation is expected to precede a shell thickening regime. When a critical temperature is not defined as above, the added resistance to moisture caused by the formation of a jammed shell layer in the thickening regime is not accounted for c.f. Section 6.2.1 [19]. It is indeed clear from Figure 15 that a shell thickening regime exist since a sharp transition from droplet drying to particle drying is not observed as in the case of drying aqueous mannitol droplet (Figure 7). Figure 16 shows the evolution

of the liquid phase mass fractions. The result indicates a high mass fraction of mannitol at the droplet surface. This can be rationalised to be the effect of the reduced diffusion rate of the solute molecules resulting from the increased solution viscosity (i.e. $D \propto \frac{1}{\eta}$ ). Nevertheless, the mass fraction of both solutes are seen to be highest at the droplet surface at all times, as expected. The droplet shows a similar temperature profile to the binary system Figure 9. Figure 17a further shows the drying histories obtained for different starting concentrations of PVP, while Figure 17b shows the corresponding relative concentration of the components at the surface. Similar to the binary system, shorter drying times and larger particle sizes are obtained with increasing PVP concentration. Figure 18 shows the evolution of 

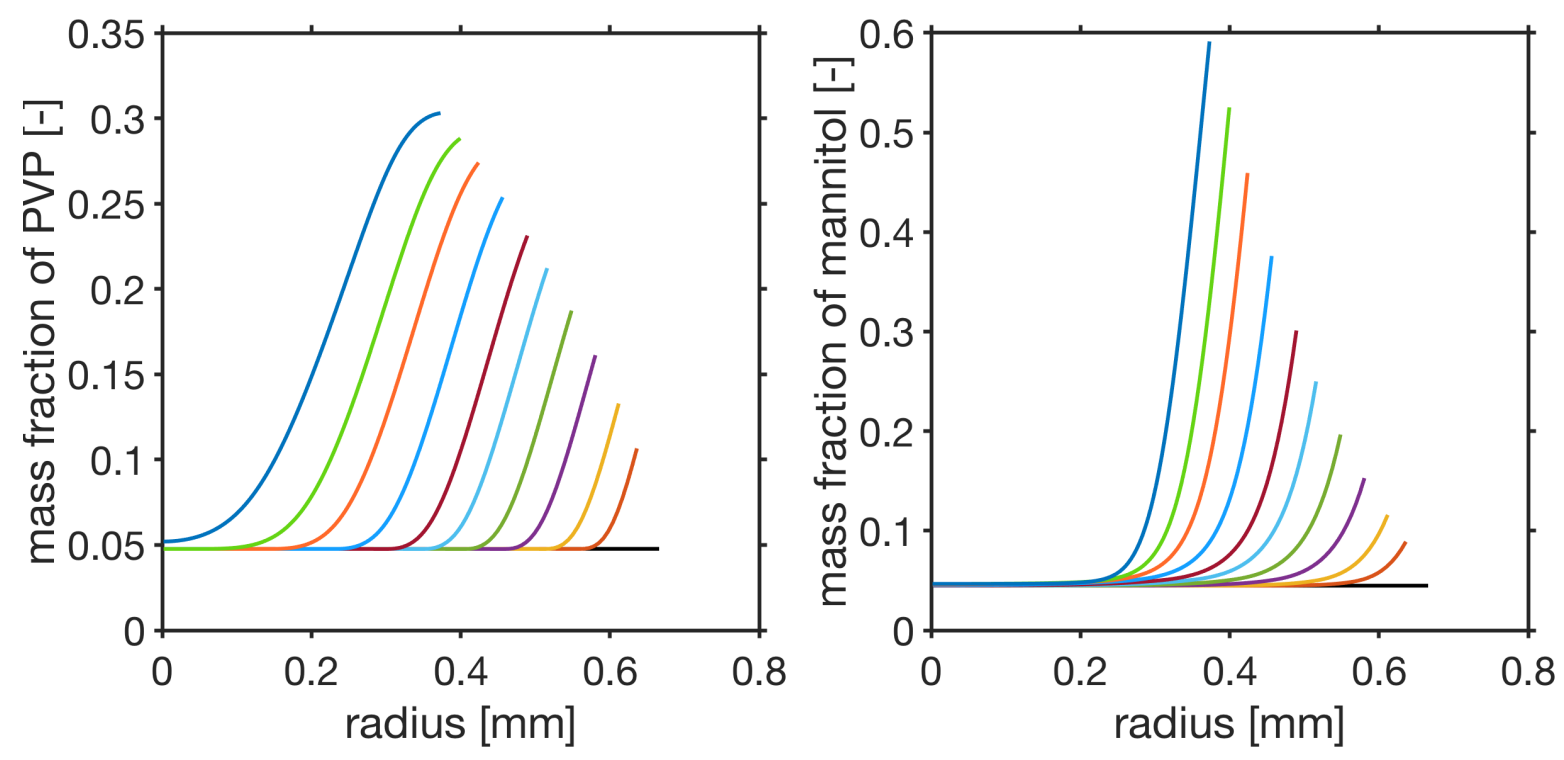

Figure 16: Model and experimental results for the drying of a droplet containing an aqueous solution of PVP and D-mannitol, $4.8 \% \mathrm{w} / \mathrm{w} \mathrm{PVP}, 4.5 \% \mathrm{w} / \mathrm{w}$ mannitol, $333 \mathrm{~K}$. This shows the mass fraction of mannitol in the liquid (left) and the mass fraction of PVP in the liquid (right).

the total number of mannitol particles within the drying droplet. As expected, this follows a similar profile to the case of mannitol-water solution (Figure 11) albeit the total number of particles is much lower than that of the binary case due to the reduced kinetics resulting from the increasing solution viscosity. This is further illustrated by the reduced solid fraction (Figure 19) at the droplet surface at the time of shell formation, indicating the dominating effect of the polymer gelation. Note that in this figure, the high mass fraction of mannitol seen will decrease significantly as drying proceeds following shell formation and the density of mannitol particles increases. The final polymer concentration at the point of shell formation is $\sim 35 \% \mathrm{w} / \mathrm{w}$, a value lower than that of the binary PVP-water system. As discussed previously, this highlights that the critical point for the ternary system considered here is not equivalent to either critical points of the binary systems (i.e. PVP-water and mannitol-water). The SEM image shown in Figure 20 further supports this claim, as the shell is visually observed to consist of crystals, while the number of crystals is significantly reduced when compared to the binary system Figure 12. 


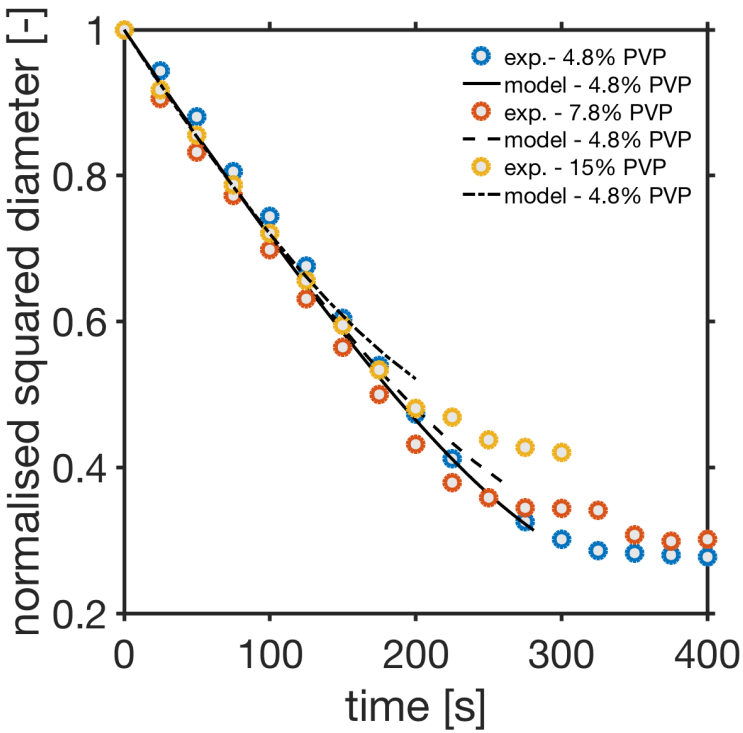

(a)

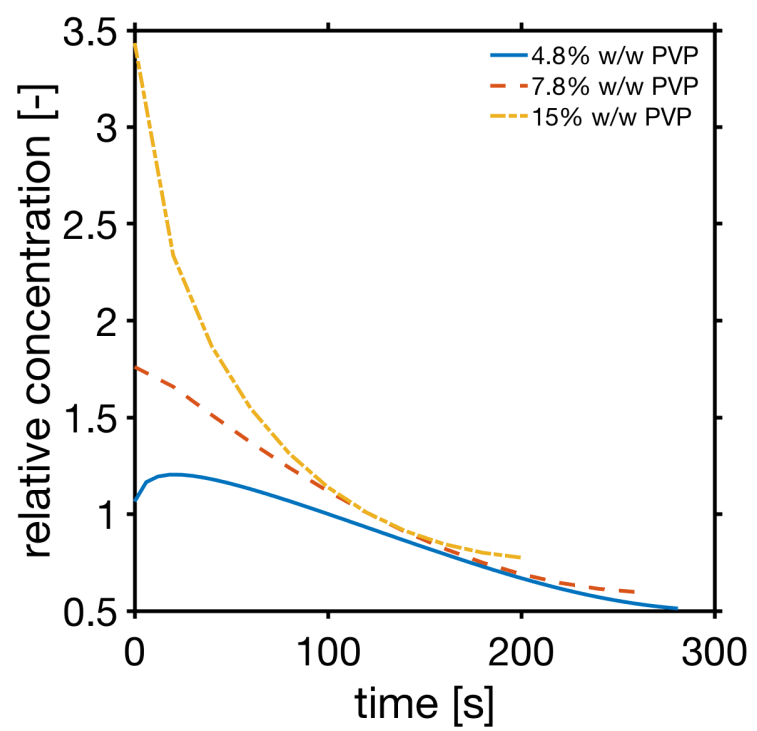

(b)

Figure 17: Application of the model to droplets with different starting PVP concentrations in 4.5\% w/w mannitol and drying at $333 \mathrm{~K} \mathrm{a}$ ) evolution of the droplet diameter b) evolution of the relative concentration of PVP to mannitol at the surface of the droplet.

\section{Conclusion}

A model consisting of mass, energy and population balance equations was developed for the drying of solution droplets. The drying history of the droplet until the point of shell formation was described through the coupling of mass balances over the liquid phase, the solid phase and internal/external energy transport. By considering case studies involving water, we show that the current model provides a sophisticated description of the history of droplets drying in a controlled environment. As illustrated from the model case studies, such a mechanistic description can be adapted to glass forming formulations (amorphous systems) if diffusion coefficients can be estimated within an acceptable level of accuracy. The use of UNIFAC group contribution method to describe the non-ideality of the liquid phase in mannitol and PVP-mannitol solutions is shown to be appropriate for the cases considered. Nevertheless, for other mixtures different activity coefficient models might be necessary. The simulation of droplet temperature profile shows that the temperature is near-uniform at all times during the first drying stage, and agrees well with available literature values of wet bulb temperatures. However, for the second drying stage temperature gradients are likely to be more pronounced, so that from a perspective of model consistency, considering the gradients already in the first drying stage is prudent. 


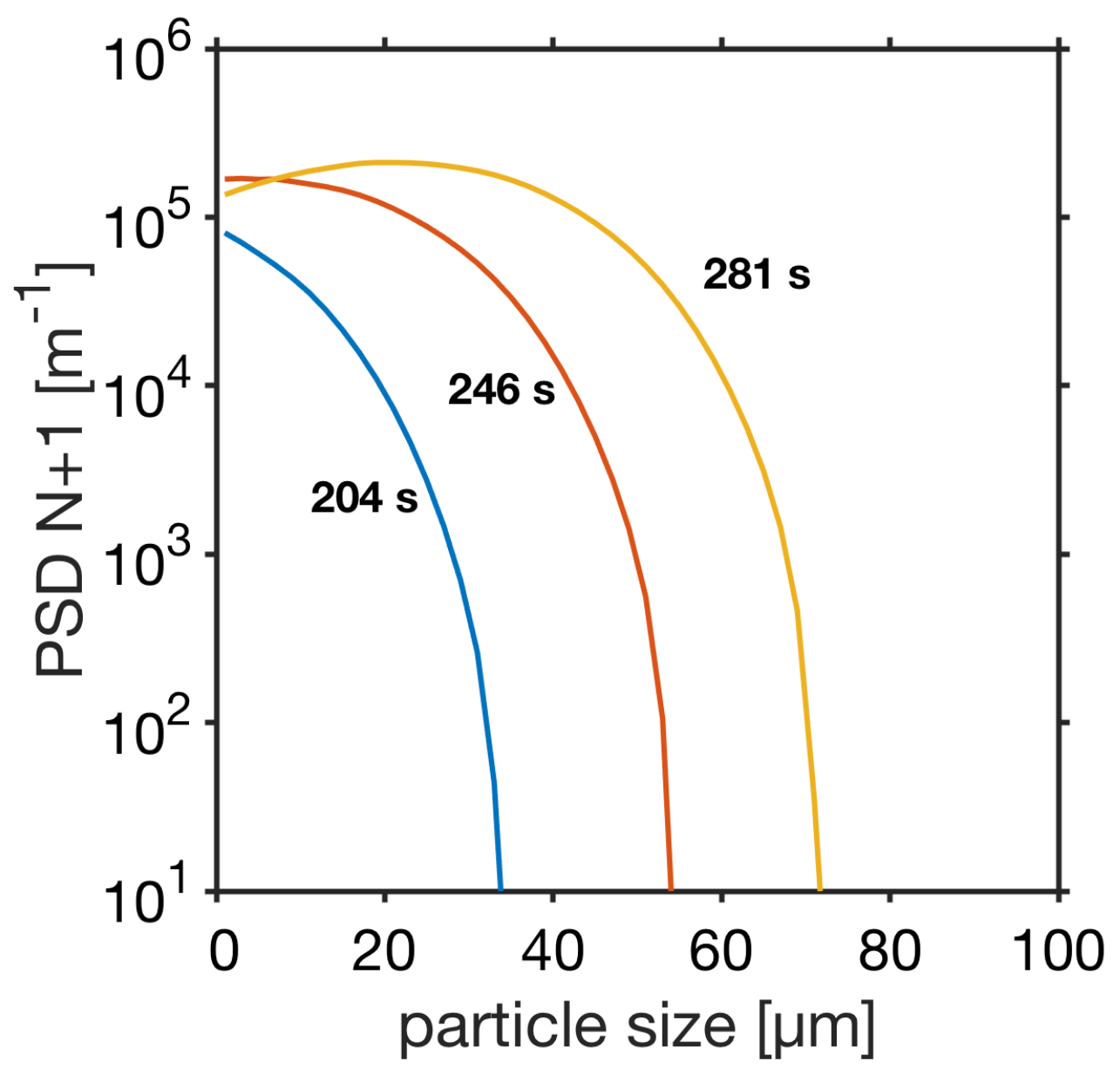

Figure 18: The evolution of the total number density of particles in the droplet during the drying of an aqueous solution of PVP and mannitol; $4.8 \%$ w/w PVP, $4.5 \%$ w/w mannitol at $333 \mathrm{~K}$.

The time of shell formation and diameter for mannitol and PVP-mannitol solutions are predicted within an acceptable margin of error. In the case of a mannitol solution, the effect of the initial droplet concentration and temperature was investigated. The results show that as the concentration is increased from approximately $1 \% \mathrm{w} / \mathrm{w}$ to $11 \% \mathrm{w} / \mathrm{w}$, the drying times become shorter and larger particles sizes are obtained, as expected. Similarly, increasing the temperature led to shorter drying times however, no significant trend was observed for the particle size. The model when applied to a more complex formulation involving a crystalline solute, polymer and a solvent shows excellent predictability and further demonstrates the capability to describe the drying behaviour of such mixtures as well. Whilst the mechanistic description of transport within a droplet shown here represents a step towards predicting particle morphologies, the drying process following shell formation needs to be further described for that to be possible. This work represents a vital contribution towards being able to predict particle 


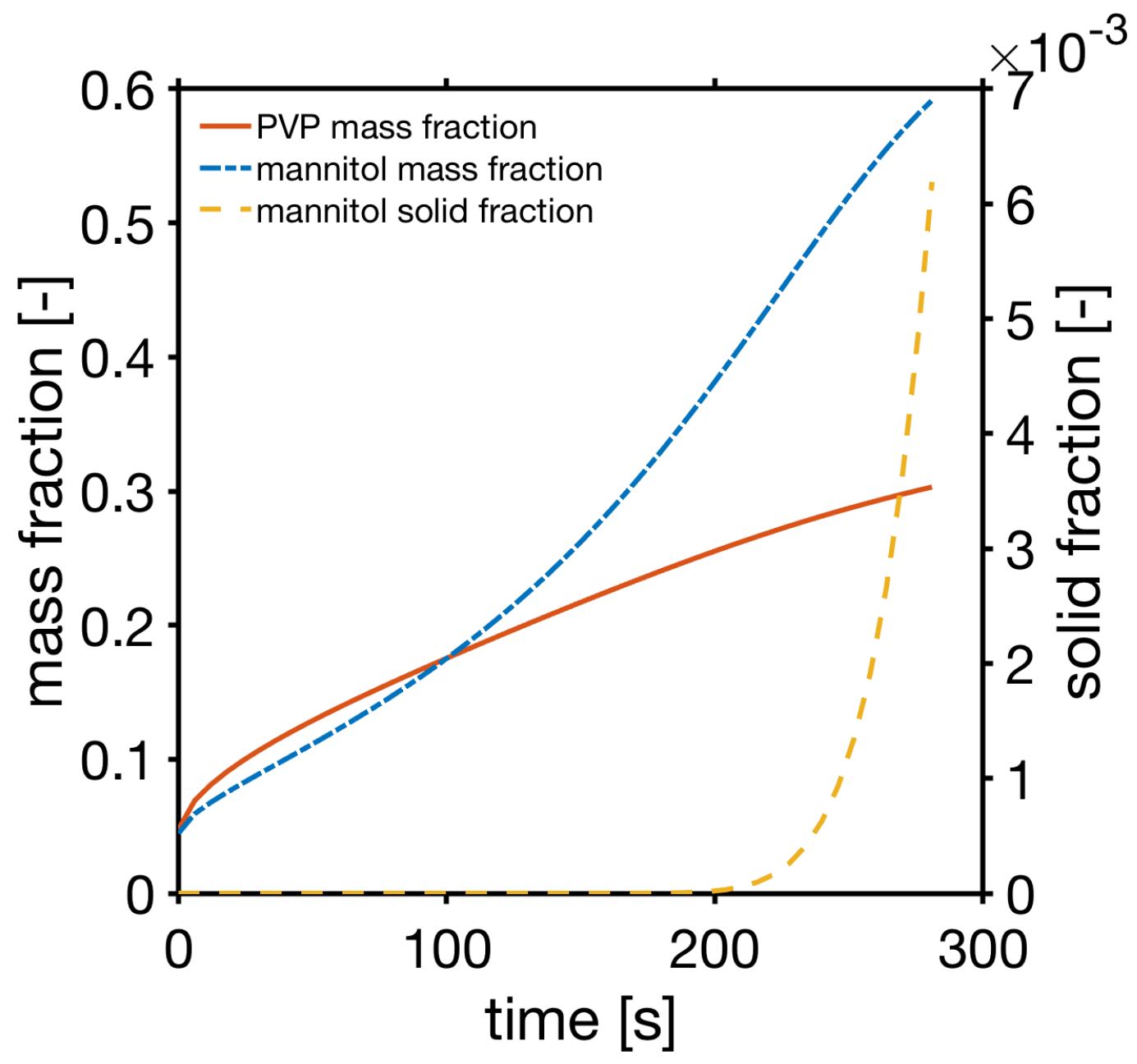

Figure 19: evolution of solute mass fractions and the solid fraction of mannitol at the droplet surface during the drying of a droplet containing an aqueous solution of PVP and mannitol; $4.8 \% \mathrm{w} / \mathrm{w}$ PVP, $4.5 \% \mathrm{w} / \mathrm{w}$ mannitol at $333 \mathrm{~K}$.

morphologies and the PSD obtained also has great potential for pharmaceutical delivery applications. 


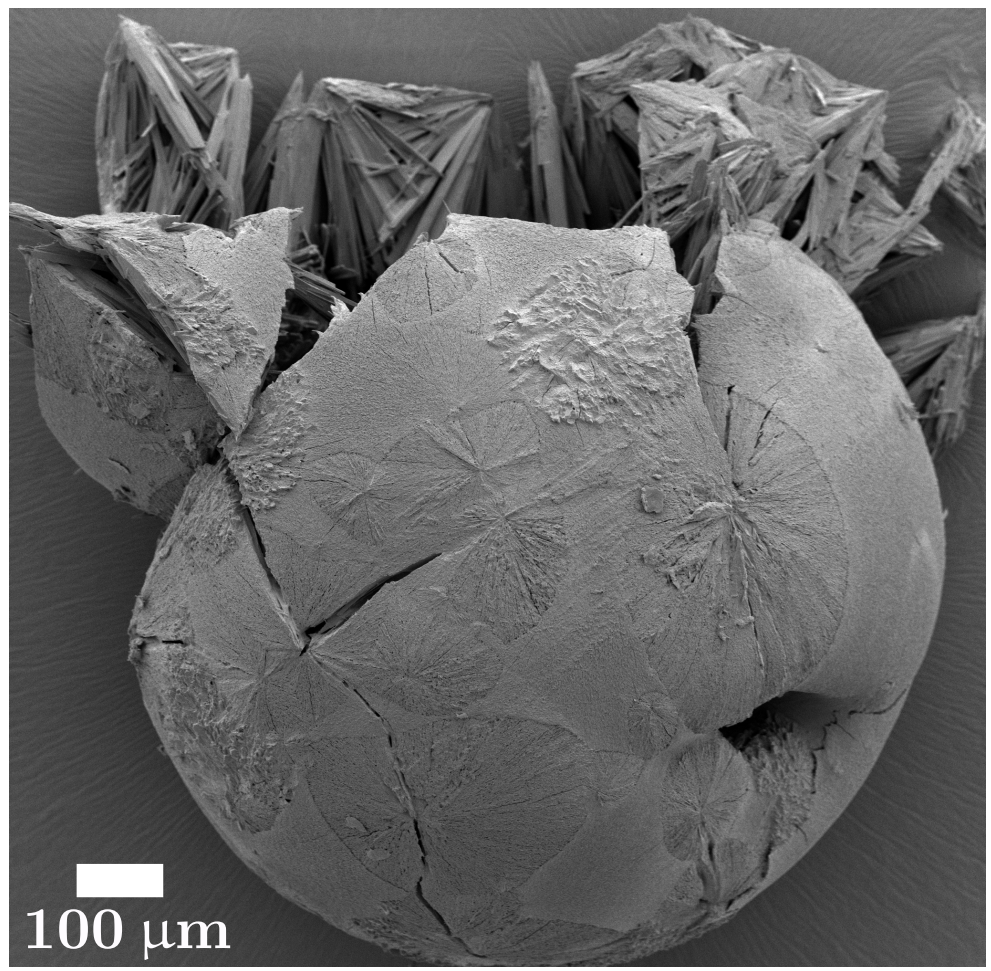

Figure 20: SEM image of a particle manufactured from a droplet with initial concentration of $4.8 \% \mathrm{w} / \mathrm{w}$ PVP in $4.5 \% \mathrm{w} / \mathrm{w}$ mannitol in water, and dried at $313 \mathrm{~K}$ and $0 \%$ relative saturation. The number of crystals formed within is observed to decrease significantly in the presence of PVP when compared to the case of mannitol-water shown in Figure 12.

\section{Acknowledgments}

This work was supported by the Eli Lilly Research Award Program (LRAP).

\section{Abbreviations}

API active pharmaceutical ingredient

CEM controlled evaporator mixer

LFC liquid flow controller

GFC gas flow controller

PBE population balance equations

PSD particle size distribution

PVP polyvinylpyrolidone 
SDD single droplet drying

627 UNIFAC UNIQUAC functional group activity coefficients

628 
[1] W. D. Callister Jr, D. G. Rethwisch, Materials Science and Engineering: An introduction, John Wiley \& Sons, Inc, New York, 9th edn., ISBN 978-1-118-47770-0, 2014.

[2] B. Jonas, Engineering pharmaceutical materials; Modulation of particle structural properties, solid state stability and tabletting behavior by drying process (Doctoral dissertation), Tech. Rep., Acta Universitatis Upsaliensis, Uppsala, Sweden, doi:ISBN91-554-5572-7, 2003.

[3] M. Davis, G. Walker, Recent strategies in spray drying for the enhanced bioavailability of poorly water-soluble drugs, Journal of Controlled Release 269 (2018) 110-127, ISSN 18734995, doi: 10.1016/j.jconrel.2017.11.005.

[4] A. K. El-Zhry El-Yafi, H. El-Zein, Technical crystallization for application in pharmaceutical material engineering: Review article, Asian Journal of Pharmaceutical Sciences 10 (4) (2014) 283-291, ISSN 2221285X, doi:10.1016/j.ajps.2015.03.003.

[5] S. V. Jermain, C. Brough, R. O. Williams, Amorphous solid dispersions and nanocrystal technologies for poorly water-soluble drug delivery - An update, International Journal of Pharmaceutics 535 (2018) 379-392, ISSN 18733476, doi:10.1016/j.ijpharm.2017.10.051.

[6] J. S. Hwang, S. H. Kim, S. H. Cho, K. M. Huh, Preparation and characterization of solid dispersions of eprosartan using spray drying method, Polymer (Korea) 37 (4) (2013) 442-448, ISSN 0379153X, doi:10.7317/pk.2013.37.4.442.

[7] A. Paudel, Z. A. Worku, J. Meeus, S. Guns, G. Van Den Mooter, Manufacturing of solid dispersions of poorly water soluble drugs by spray drying: Formulation and process considerations, International Journal of Pharmaceutics 453 (1) (2013) 253-284, ISSN 18733476, doi: 10.1016/j.ijpharm.2012.07.015.

[8] J. Patil, Spray-Drying: An Emerging Technique for Pharmaceutical Product Development, Journal of Pharmacovigilance 04 (02) (2016) 10-11, ISSN 23296887, doi:10.4172/2329-6887. $1000 \mathrm{e} 150$

[9] J. A. Champion, Y. K. Katare, S. Mitragotri, Particle shape: A new design parameter for micro- 
and nanoscale drug delivery carriers, Journal of Controlled Release 121 (1-2) (2007) 3-9, ISSN 01683659, doi:10.1016/j.jconrel.2007.03.022.

[10] D. E. Walton, C. J. Mumford, Spray dried products-characterization of particle morphology, Chemical Engineering Research and Design 77 (1) (1999) 21-38, ISSN 02638762, doi:10.1205/ 026387699525846 .

[11] N. Fu, M. W. Woo, X. D. Chen, Single Droplet Drying Technique to Study Drying Kinetics Measurement and Particle Functionality: A Review, Drying Technology 30 (15) (2012) 17711785, ISSN 07373937, doi:10.1080/07373937.2012.708002.

[12] M. Mezhericher, A. Levy, I. Borde, Theoretical drying model of single droplets containing insoluble or dissolved solids, Drying Technology 25 (6) (2007) 1025-1032, ISSN 07373937, doi: $10.1080 / 07373930701394902$.

[13] A. L. Yarin, M. Pfaffenlehner, C. Tropea, On the acoustic levitation of droplets, Journal of Fluid Mechanics 356 (1998) 65-91, ISSN 00221120, doi:10.1017/S0022112097007829.

[14] A. L. Yarin, G. Brenn, O. Kastner, D. Rensink, C. Tropea, Evaporation of acoustically levitated droplets, Journal of Fluid Mechanics 399 (1999) 151-204, ISSN 00221120, doi:10.1017/ S0022112099006266.

[15] A. L. Yarin, G. Brenn, O. Kastner, C. Tropea, Drying of acoustically levitated droplets of liquidsolid suspensions: Evaporation and crust formation, Physics of Fluids 14 (7) (2002) 2289-2298, ISSN 10706631, doi:10.1063/1.1483308.

[16] O. Kastner, G. Brenn, D. Rensink, C. Tropea, The acoustic tube levitator - A novel device for determining the drying kinetics of single droplets, Chemical Engineering and Technology 24 (4) (2001) 335-339, ISSN 09307516, doi:10.1002/1521-4125(200104)24:4〈335::AID-CEAT335〉3. $0 . \mathrm{CO} ; 2-8$.

[17] A. Osman, L. Goehring, A. Patti, H. Stitt, N. Shokri, Fundamental Investigation of the Drying of Solid Suspensions, Industrial and Engineering Chemistry Research 56 (37) (2017) 10506-10513, ISSN 15205045, doi:10.1021/acs.iecr.7b02334. 
[18] M. Mezhericher, A. Levy, I. Borde, Theoretical models of single droplet drying kinetics: A review, Drying Technology 28 (2) (2010) 278-293, ISSN 07373937, doi:10.1080/07373930903530337.

[19] C. S. Handscomb, M. Kraft, A. E. Bayly, A new model for the drying of droplets containing suspended solids, Chemical Engineering Science 64 (4) (2009) 628-637, ISSN 00092509, doi: 10.1016/j.ces.2008.04.051

[20] C. Handscomb, M. Kraft, A. Bayly, A new model for the drying of droplets containing suspended solids after shell formation, Chemical Engineering Science 64 (2) (2009) 228-246, ISSN 00092509, doi:10.1016/j.ces.2008.10.019.

[21] P. Seydel, J. Blömer, J. Bertling, Modeling particle formation at spray drying using population balances, Drying Technology 24 (2) (2006) 137-146, ISSN 07373937, doi:10.1080/ 07373930600558912 .

[22] C. S. Handscomb, M. Kraft, Simulating the structural evolution of droplets following shell formation, Chemical Engineering Science 65 (2) (2010) 713-725, ISSN 00092509, doi:10.1016/j. ces.2009.09.025.

[23] M. Farid, A new approach to modelling of single droplet drying, Chemical Engineering Science 58 (13) (2003) 2985-2993, ISSN 00092509, doi:10.1016/S0009-2509(03)00161-1.

[24] W. E. Ranz, W. Marshall, Evaporation from drops, Chemical Engineering Progress 48 (3) (1952) $141-180$.

[25] D. H. Charlesworth, W. R. Marshall, Evaporation from drops containing dissolved solids, AIChE Journal 6 (1) (1960) 9-23, ISSN 15475905, doi:10.1002/aic.690060104.

[26] S. Nešić, J. Vodnik, Kinetics of droplet evaporation, Chemical Engineering Science 46 (2) (1991) 527-537, ISSN 00092509, doi:10.1016/0009-2509(91)80013-O.

[27] S. R. Gopireddy, E. Gutheil, Numerical simulation of evaporation and drying of a bi-component droplet, International Journal of Heat and Mass Transfer 66 (2013) 404-411, ISSN 00179310, doi:10.1016/j.ijheatmasstransfer.2013.07.010. 
[28] D. E. Walton, The morphology of spray-dried particles a qualitative view, Drying Technology 18 (9) (2000) 1943-1986, ISSN 07373937, doi:10.1080/07373930008917822.

[29] E. L. Cussler, Diffusion: Mass Transfer in Fluid Systems, Cambridge University press, New York, third edn., ISBN 978-0-511-47892-5, doi:10.1017/CBO9780511805134.010, 2009.

[30] B. Abramzon, W. A. Sirignano, Droplet vaporization model for spray combustion calculations, International Journal of Heat and Mass Transfer 32 (9) (1989) 1605-1618, ISSN 00179310, doi: 10.1016/0017-9310(89)90043-4.

[31] G. Brenn, L. J. Deviprasath, F. Durst, C. Fink, Evaporation of acoustically levitated multicomponent liquid droplets, International Journal of Heat and Mass Transfer 50 (25-26) (2007) 5073-5086, ISSN 00179310, doi:10.1016/j.ijheatmasstransfer.2007.07.036.

[32] A. Acrivos, Bubbles, Drops and Particles, vol. 94, Academic Press, New York, ISBN 012176950X, doi:10.1017/s0022112079221290, 1979.

[33] S. S. Sazhin, A. E. Elwardany, P. A. Krutitskii, V. Deprédurand, G. Castanet, F. Lemoine, E. M. Sazhina, M. R. Heikal, Multi-component droplet heating and evaporation: Numerical simulation versus experimental data, International Journal of Thermal Sciences 50 (7) (2011) 1164-1180, ISSN 12900729, doi:10.1016/j.ijthermalsci.2011.02.020.

[34] I. M. Smallwood, Handbook of Organic Solvent Properties, Handbook of Organic Solvent Properties 17 (2) (2012) 1-306, ISSN 01437496, doi:10.1016/C2009-0-23646-4.

[35] E. B. Poling, M. J. Prausnitz, P. O'Connell, The properties of Liquids and Gases, McGraw Hill, Chicago, 5th edn., ISBN 0071499997, doi:10.1036/0070116822, 2001.

[36] H. Y. Sohn, C. Moreland, The effect of particle size distribution on packing density, The Canadian Journal of Chemical Engineering 46 (3) (1968) 162-167, ISSN 1939019X, doi:10.1002/cjce. 5450460305 .

[37] G. Perini, F. Salvatori, D. R. Ochsenbein, M. Mazzotti, T. Vetter, Filterability prediction of needlelike crystals based on particle size and shape distribution data, Separation and Purification Technology 211 (2019) 768-781, ISSN 18733794, doi:10.1016/j.seppur.2018.10.042. 
[38] A. P. Philipse, The Random Contact Equation and Its Implications for (Colloidal) Rods in Packings, Suspensions, and Anisotropic Powders, Langmuir 12 (24) (1996) 5971-5971, ISSN 07437463, doi:10.1021/la960869o.

[39] J. C. Strikwerda, Book Review: Numerical methods for conservation laws, Bulletin of the American Mathematical Society 28 (2) (2008) 370-374, ISSN 0273-0979, doi:10.1090/ s0273-0979-1993-00366-5.

[40] B. Koren, A robust upwind discretization method for advection, diffusion and source terms, in: C.B. Vreugdenhil \& B. Koren (Eds.), Numerical Methods for Advection-Diffusion Problems, vol. 45, chap. 5, Centrum voor Wiskunde en Informatica, Amsterdam, ISBN 9783528076450, 117-138, 1993.

[41] L. F. Shampine, M. W. Reichelt, The MATLAB ode suite, SIAM Journal of Scientific Computing 18 (1) (1997) 1-22, ISSN 10648275, doi:10.1137/S1064827594276424.

[42] G. A. E. Godsave, Studies of the combustion of drops in a fuel spray-the burning of single drops of fuel, Symposium (International) on Combustion 4 (1) (1953) 818-830, ISSN 00820784, doi: 10.1016/S0082-0784(53)80107-4.

[43] D. B. Spalding, The combustion of liquid fuels, Symposium (International) on Combustion 4 (1) (1953) 847-864, ISSN 00820784, doi:10.1016/S0082-0784(53)80110-4.

[44] S. K. Aggarwal, A. Y. Tong, W. A. Sirignano, A comparison of vaporization models in spray calculations, AIAA Journal 22 (10) (1984) 1448-1457, ISSN 00011452, doi:10.2514/3.8802.

[45] D. C. Shallcross, Psychrometric charts for hydrocarbon vapours in nitrogen, Calphad: Computer Coupling of Phase Diagrams and Thermochemistry 20 (3) (1996) 273-288, ISSN 03645916, doi: $10.1016 / \mathrm{S} 0364-5916(96) 00030-2$.

[46] D. C. Shallcross, Preparation of Psychrometric Charts for Alcohol Vapours in Nitrogen, Korean Journal of Chemical Engineering 17 (1) (2000) 93-100, ISSN 02561115, doi:10.1007/ BF02789260. 
[47] M. A. Silva, P. J. Kerkhof, W. J. Coumans, Estimation of effective diffusivity in drying of heterogeneous porous media, Industrial and Engineering Chemistry Research 39 (5) (2000) 1443-1452, ISSN 08885885, doi:10.1021/ie990563n.

[48] H. L. Ohrem, E. Schornick, A. Kalivoda, R. Ognibene, Why is mannitol becoming more and more popular as a pharmaceutical excipient in solid dosage forms?, Pharmaceutical Development and Technology 19 (3) (2014) 257-262, ISSN 10979867, doi:10.3109/10837450.2013.775154.

[49] J. Cornel, P. Kidambi, M. Mazzotti, Precipitation and transformation of the three polymorphs of d-mannitol, Industrial and Engineering Chemistry Research 49 (12) (2010) 5854-5862, ISSN 08885885, doi:10.1021/ie9019616.

[50] S. R. Werner, R. L. Edmonds, J. R. Jones, J. E. Bronlund, A. H. Paterson, Single droplet drying: Transition from the effective diffusion model to a modified receding interface model, Powder Technology 179 (3) (2008) 184-189, ISSN 00325910, doi:10.1016/j.powtec.2007.06.009.

[51] A. Porowska, M. Dosta, L. Fries, A. Gianfrancesco, S. Heinrich, S. Palzer, Predicting the surface composition of a spray-dried particle by modelling component reorganization in a drying droplet, Chemical Engineering Research and Design 110 (2016) 131-140, ISSN 02638762, doi:10.1016/ j.cherd.2016.03.007.

[52] Z. A. Metaxiotou, S. G. Nychas, Experimental measurement of diffusion in aqueous polyvinylpyrrolidone solutions, AIChE Journal 41 (4) (1995) 812-818, ISSN 15475905, doi: 10.1002/aic.690410408.

[53] D. Kashchiev, Nucleation: Basic Theory with Applications, Butterworth-Heinemann, Oxford, ISBN 9780080537832, doi:10.1088/0031-9120/15/2/102, 2000.

[54] M. Ohara, R. Reid, Modelling Crystal Growth Rates from Solution, Prentice Hall,, Englewood Cliffs, New Jersey, ISBN 0135861314, 1973.

[55] D. Bolten, M. Türk, Experimental study on the surface tension, density, and viscosity of aqueous poly(vinylpyrrolidone) solutions, Journal of Chemical and Engineering Data 56 (3) (2011) 582 588, ISSN 00219568, doi:10.1021/je101277c. 
[56] Ashland, PVP - Polyvinylpyrolidone polymers (Brochure), https://www.brenntag.com/media/ documents/bsi/product_data_sheets/material_science/ashland_polymers/pvp_polymers_brochure. pdf, last visited 15-03-2020, 2013. 